\title{
1 Approaches to integrating genetic data into ecological networks
}

3 Running Head: Molecular Food Webs

5 Elizabeth L. Clare ${ }^{1,2}$, Aron J. Fazekas ${ }^{3}$, Natalia V. Ivanova ${ }^{2}$, Robin M. Floyd ${ }^{4}$, Paul D.N.

6 Hebert $^{2}$, Amanda M. Adams ${ }^{5}$, Juliet Nagel ${ }^{6}$, Rebecca Girton ${ }^{1}$, Steven G. Newmaster ${ }^{3}$, M.

$7 \quad$ Brock Fenton ${ }^{7}$

$9 \quad{ }^{1}$ School of Biological and Chemical Sciences, Queen Mary University of London.

10 London UK. E14NS

$11{ }^{2}$ Centre for Biodiversity Genomics, University of Guelph, Guelph Ontario, Canada N1G

$122 \mathrm{~W} 1$

$13{ }^{3}$ Biodiversity Institute of Ontario, University of Guelph, Guelph Ontario, Canada N1G

$14 \quad 2 \mathrm{~W} 1$

$15 \quad{ }^{4}$ Welcome Trust Stem Cell Institute, University of Cambridge, Cambridge, UK

$16{ }^{5}$ Department of Biology, Texas A\&M University, 3258 TAMU, College Station 77843

17 USA

$18{ }^{6}$ University of Maryland, Center for Environmental Science, Frostburg, MD, USA

$19{ }^{7}$ Department of Biology, University of Western Ontario, London, ON N6A 5B7, Canada

21 Corresponding Author: Elizabeth Clare, School of Biological and Chemical Sciences,

22 Queen Mary University of London. London UK. E14NS, e.clare@qmul.ac.uk 


\section{Abstract}

25 As molecular tools for assessing trophic interactions become common, research is

26 increasingly focused on the construction of interaction networks. Here we demonstrate

27 three key methods for incorporating DNA data into network ecology and discuss

28 analytical considerations using a model consisting of plants, insects, bats and their

29 parasites from the Costa Rican dry forest. The simplest method involves the use of

30 Sanger sequencing to acquire long sequences to validate or refine field identifications, for

31 example of bats and their parasites, where one specimen yields one sequence and one

32 identification. This method can be fully quantified and resolved and these data resemble

33 traditional ecological networks. For more complex taxonomic identifications, we target

34 multiple DNA loci e.g. from a seed or fruit pulp sample in faeces. These networks are

35 also well resolved but gene targets vary in resolution and quantification is difficult.

36 Finally for mixed templates such as faecal contents of insectivorous bats we use DNA

37 metabarcoding targeting two sequence lengths (157bp, 407bp) of one gene region and a

38 MOTU, BLAST and BIN association approach to resolve nodes. This network type is

39 complex to generate and analyse and we discuss the implications of this type of

40 resolution on network analysis. Using these data we construct the first molecular-based

41 network of networks containing 3304 interactions between 762 nodes of 8 trophic

42 functions and involving parasitic, mutualistic, and predatory interactions. We provide a

43 comparison of the relative strengths and weaknesses of these data types in network

44 ecology.

45 Key Words: food webs, interaction networks, DNA barcoding, metabarcoding, high-

46 throughput sequencing, bats 


\section{Introduction:}

\section{Ecological Networks, DNA \& Opportunities}

49 Ecosystem functioning is driven by a network of interactions among species

50 affected by diverse abiotic and biotic variables such as climate, habitat, and resource

51 distribution (McCann, 2007) with global economic (Costanza et al., 1997) and

52 conservation (Worm et al., 2006) impacts. The analysis of interaction networks is of

53 increasing interest across many disciplines, spurring the development of new

54 mathematical and statistical tools (Poisot, Stouffer, \& Kéfi, 2016). In ecology, visual

55 representations provide a synoptic view of complex interactions and are primarily

56 displayed as bipartite networks, where trophic levels are depicted as layers (upper and

57 lower) composed of species as nodes connected by links representing interactions

58 (Dormann, Fründ, Blüthgen, \& Gruber, 2009). When multiple networks are combined, it

59 is possible to conceptualise multiple trophic levels simultaneously (e.g., Pocock, Evans

60 and Memmott 2012) clarifying ecosystem assembly and structure (Milo et al., 2002),

61 functional roles, and mechanisms of stability (McCann, 2000; Thébault \& Fontaine,

62 2010). Comparisons between networks can assess natural or anthropogenic impacts

63 (McCann, 2007), the evolution of networks (Guimarães Jr, Jordano, \& Thompson, 2011;

64 Nuismer, Jordano, \& Bascompte, 2013) and the role and response of specific nodes

65 (Martín González, Dalsgaard, \& Olesen, 2010; McDonald-Madden et al., 2016; Strona \& 66 Lafferty, 2016).

67 Many networks are incredibly complex with multiple trophic levels and high

68 taxonomic diversity (e.g. Pocock et al. 2012), and are therefore time consuming to

69 construct, often requiring years of ecological observations and considerable taxonomic 
70 expertise (Evans, Kitson, Lunt, Straw, \& Pocock, 2016). Consequently, they are not

71 readily scalable to rapid bio-monitoring or, if they can scale, they routinely suffer from

72 problems of network completeness and poor or uneven resolution of taxa (Hemprich-

73 Bennett, Oliveira, Le Comber, Rossiter, \& Clare, 2018; Ings et al., 2009). Incorporating

74 dietary tracers such as fatty acids, isotopes, and genetic tools such as DNA sequencing is

75 a growing trend for measuring species interactions, though each has advantages and

76 disadvantages (reviewed in Nielsen, Clare, Hayden, Brett, \& Kratina, 2018). Genetic

77 analyses are expanding at a remarkable rate and have evolved from enzyme-linked

78 immunosorbent assay (ELISA) and targeted species detection (Symondson, 2002) to the

79 use of high-throughput sequencing (HTS) for the analysis of target markers or

80 "metabarcoding" (reviewed in Pompanon et al., 2012). While these techniques are

81 quickly becoming common for the dietary analysis of single species with many proposed

82 applications (Clare, 2014), they have not been widely incorporated into network analysis

83 (but see González-Varo, Arroyo, \& Jordano, 2014; Hemprich-Bennett et al. 2018; Wirta

84 et al., 2014) despite strong advocates (Evans et al., 2016; Roslin \& Majaneva, 2016).

85 Many reviews, authors, and developers of these techniques have discussed the

86 challenges in DNA-based analyses of species interactions including the impacts of primer

87 choice on taxonomic coverage and resolution, the completeness of reference databases

88 (Pompanon et al., 2012), bioinformatics methods (Clare, Chain, Littlefair, \& Cristescu,

89 2016) and the role of quantification (Deagle et al., 2018) but the specific implications for

90 constructing networks vary with data type. In traditional DNA barcoding a specimen's ID

91 is delimited by generating one sequence per specimen and comparing it to a reference

92 dataset to confirm its identity. These data are not fundamentally different from traditional 
93 observations for generating an interaction matrix. However, at the other end of the

94 complexity spectrum, metabarcoding represents a novel data type for network ecology.

95 HTS generates millions of sequences from each sample of mixed template. While the

96 process can uniquely deal with otherwise intractable sources such as trace material and

97 liquid feeders, it poses novel problems for ecological analysis and network ecology. First,

98 the data require complex bioinformatics handling to remove unwanted (often error prone)

99 data, but in many cases the impacts of these parameter choices on ecological analysis are

100 unknown (Clare et al., 2016). Second, the ability to quantify DNA within a sample is

101 highly controversial and while, in some cases, proportions of recovered sequence

102 correspond to biomass, in other cases the connection is not clear (Deagle et al., 2018;

103 Deagle, Thomas, Shaffer, Trites, \& Jarman, 2013; Thomas, Deagle, Eveson, Harsch, \&

104 Trites, 2016). Finally, in an ideal situation, the recovered sequences are matched to a

105 complete reference dataset to identify taxa, but in most cases the reference library is

106 incomplete. In these cases either an incomplete network is created biased towards the

107 contents of the reference collection (often larger, more charismatic or economically

108 important taxa) or the recovered pool of DNA is converted into molecular operational

109 taxonomic units - MOTUs (Floyd, Abebe, Papert, \& Blaxter, 2002) - which are best

110 viewed as pools of equivalent genetic diversity that can be compared, whether we know

111 their identity or not (Clare et al., 2016; Floyd et al., 2002). In this case, each MOTU

112 becomes a node in the network and this level of the network is entirely resolved to a

113 common point of reference with both known and unknown items included, a distinct

114 advantage when mixed resolution presents an analytical problem (Hemprich-Bennett et

115 al., 2018; Ings et al., 2009). However, the level of this resolution is arbitrarily defined by 
116 the bioinformatics assessment (see an analysis of parameters for MOTU definition in

117 ecological analysis (Clare et al., 2016; Flynn, Brown, Chain, MacIsaac, \& Cristescu,

118 2015) which may collapse trophic levels and thus generate fundamentally different

119 structures. This is of importance when selecting what metrics can or should be measured.

120 Networks metrics can be divided into several broad classes. Network level metrics (e.g.

121 connectance, nestedness, generality) are measured across the entire network. Node level

122 metrics (e.g. centrality, species strength, partner diversity) are specific to the interactions

123 of a given node. Motif measurements are sub-network of a particular pattern which may

124 define specific ecological interaction types or functions (Milo et al., 2002). Each metric

125 type needs to be considered separately in light of the new data type. Many have

126 concluded that the molecular approach will transform the discipline of ecological

127 biomonitoring and ecological network analysis permitting rapid consistent assessments in

128 systems that are otherwise intractable (Gibson et al., 2015; Roslin \& Majaneva, 2016;

129 Toju, 2015; Wirta et al., 2014) while others have advocated adoption but raised serious

130 analytical concerns (e.g. Evans et al., 2016). How then might we proceed?

131 Here we address this challenge by focussing on a single complex assemblage of

132 interacting species to demonstrate three approaches to the use of DNA data to resolve

133 interactions and measure several network and node level metrics. While these are not

134 without controversy, our objective is to present an example of methods of data integration

135 into a "network of networks" and we include the most commonly analysed interaction

136 types (antagonistic, mutualistic, parasitic) and the three key methods that have been

137 discussed for DNA and network integration. First, we use standard single-gene DNA

138 barcoding to resolve taxonomy in cryptic organisms and to validate field identifications. 
139 Second, we use multi-gene DNA barcoding to resolve more complex taxa and single-

140 sourced trace material that cannot be identified by morphological methods. Third, we use

141 metabarcoding to resolve mixed material, and then discuss the advantages and challenges

142 of applying these approaches. While these have been used previously, our analysis

143 provides the first example of integrating these data types to form a multi-trophic level

144 assemblage resolved entirely with DNA and the first to contrast these data. We hope to

145 provoke discussion about the appropriate use of these data types.

147 Materials and Methods:

148 A case study from Cost Rica: Plants, Bats, Insects, and Parasites

149 From May to July 2009, a field team visited Sector Santa Rosa of the Area de

150 Conservación Guanacaste (ACG). The present analysis relies on material collected during

151 that period and a preliminary ecological analysis of this case is presented (Box 1). A total

152 of 801 bats were captured representing 26 species morphologically identified using

153 available field keys and checklists (Reid, 2009; Simmons, 2005). From these individuals

154 we analyzed 466 parasites that were sampled from 18 host species and 260 guano

155 samples from 21 species of which visual inspection led to 132 samples being classified as

156 containing plant materials (seeds or fruit pulp) and the rest insect material. Some species

157 are integrated into all trophic levels while others are only loosely associated, for example

158 the sanguivore Desmodus rotundus did not produce a faecal sample so was retained in the

159 dataset as a parasite host only.

160

161 Method one: Sanger sequencing to resolve species ID of bats and parasites 
163 clarify species boundaries or to confirm and improve upon identifications made in the

164 field. For bats and parasites we targeted the 5' end of the mitochondrial cytochrome $c$

165 oxidase subunit 1 gene (COI) as described by Hebert, Ratnasingham, \& DeWaard, (2003)

166 using full length (658 bp) DNA barcodes which provide taxonomic discrimination for

167 most animal groups (Hebert, Cywinska, Ball, \& DeWaard, 2003). For bats we used small

168 tissue fragments from each individual captured and the "routine" method of DNA

169 barcoding described in Ivanova, Clare, \& Borisenko, (2012) and the mammal primer

170 cocktail, PCR reagent mix and the thermocycler conditions "MamCOI" described in

171 Tables S1, S2 and S3 of that publication. We edited sequences in CodonCode Aligner

172 (CodonCode Corporation, Centerville, MA) and compared the resulting DNA barcodes to

173 existing reference databases (Clare, Lim, Fenton, \& Hebert, 2011) using a Neighbor-

174 Joining (NJ) tree to confirm they clustered with other representatives of their species

175 assignment based on morphological inspection in the field (Figure S1). Sequences,

176 collection information, and primer names are available in the Barcode of Life Data

177 System (BOLD) (www.barcodinglife.org) (Ratnasingham \& Hebert, 2007) project BCCR

178 for each recovered sequence.

179 For parasitic flies and mites, we extracted DNA from whole specimens using 180 voucher-retention procedures (Porco, Rougerie, Deharveng, \& Hebert, 2010). Our

181 subsequent PCRs used a variety of primer combinations which are associated with

182 individual records available in the project BCPB available in the BOLD website with

183 corresponding primer sequences anline at

184 http://www.boldsystems.org/index.php/Public_Primer_PrimerSearch. Our PCR protocols 
185 followed (Hebert et al., 2013) with post sequence analysis employing CodonCode

186 Aligner (CodonCode Corporation, Centerville, MA). Unlike bats, field taxonomic

187 designations were minimal and a full reference database of voucher-linked barcodes

188 was not available. As a consequence, we employed the Barcode Identification

189 Number (BIN) (Ratnasingham \& Hebert, 2013) method of delimiting MOTUs in

190 BOLD to identify species and compared this to terminal clusters in an $\mathrm{NJ}$ tree

191 generated in BOLD. Three clusters were unassigned to any BIN because their

192 sequence lengths were insufficient to provide a sequence match with high

193 confidence; therefore, we designate these as taxa based on reciprocal monophyly of

194 their sequences in an NJ tree (Figure S2).

195

196 Method two: Sanger sequencing with multiple targets

197 A more complex problem involves the analysis of material from one source when

198 that material is degraded, making DNA analysis a preferred option, but where the taxa

199 involved are difficult to resolve using this approach. In this case, the seeds defecated by

200 bats may be identifiable from morphology, but fragmented seeds and digested fruit pulp

201 are almost never identifiable morphologically. Consequently, plants whose seeds are too

202 large to be swallowed are often excluded from food webs and dietary analyses unless

203 direct observation confirms their consumption. Plants represent an additional hurdle as

204 species delimitation by DNA often requires multiple genetic markers (CBOL plant 205 working group, 2009).

206 For all guano samples containing seeds, we separated three to five intact 207 morphologically identical seeds from each sample. For samples containing only pulp or 
208 pollen we separated approximately $10 \mathrm{mg}$ of dried guano for DNA extraction. DNA 209 isolated employed the NucleoSpin ${ }^{\circledR} 96$ Plant II DNA isolation kit (Macherey-Nagel)

210 following the manufacturer's protocol with an extension of lysis to one hour.

211 We amplified the $r b c \mathrm{~L}$ and trn H-psbA regions using primers 212 rbcLa_F/rbcLajf_634R and trnH/psbA (Fazekas et al., 2008; Kress, Wurdack, Zimmer, 213 Weigt, \& Janzen, 2005). We amplified matK using primers 1R_KIM/3F_KIM (Fazekas 214 et al., 2008) and repeated the PCR for failed reactions using alternate primers:

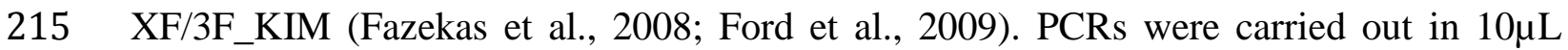
216 volumes containing $2 \mu \mathrm{L}$ of $5 \mathrm{X}$ Phire ${ }^{\circledR}$ reaction buffer (Finnzymes), $0.05 \mu \mathrm{L}$ of $10 \mathrm{mM}$ 217 dNTPs, $0.1 \mu \mathrm{L}$ of each $10 \mu \mathrm{M}$ primer, and $0.125 \mu \mathrm{L}$ of Phire ${ }^{\circledR}$ Hot Start II polymerase 218 (Finnzymes) using the following protocol: initial denaturation at $98^{\circ} \mathrm{C}$ for $90 \mathrm{~s}, 35$ cycles 219 of $98^{\circ} \mathrm{C}$ for $5 \mathrm{~s}, 55-66^{\circ} \mathrm{C}$ for $10 \mathrm{~s}$ (depending on primer set), $72^{\circ} \mathrm{C}$ for $7-10$ s (depending 220 on region), followed by a final extension at $72^{\circ} \mathrm{C}$ for 60 s and hold at $4{ }^{\circ} \mathrm{C}$ (see primer 221 references for additional details).

222 We sequenced each amplicon bi-directionally with the same primers used for 223 amplification in $11 \mu \mathrm{L}$ reaction volumes containing $0.5 \mu \mathrm{L}$ of BigDye terminator mix 224 (ABI), $2 \mu \mathrm{L}$ of $5 \mathrm{X}$ sequencing buffer, $1 \mu \mathrm{L} 10 \mathrm{uM}$ primer, and $0.5 \mu \mathrm{L}$ of undiluted $\mathrm{PCR}$ 225 product using the following protocol: initial denaturation at $96^{\circ} \mathrm{C}$ for $2 \mathrm{~min}, 30$ cycles of $22696^{\circ} \mathrm{C}$ for $30 \mathrm{~s}, 55^{\circ} \mathrm{C}$ for $15 \mathrm{~s}, 60^{\circ} \mathrm{C}$ for $4 \mathrm{~min}$, followed by a hold at $4^{\circ} \mathrm{C}$.

227 We assembled contigs and edited all sequences using Sequencher 4.8 (Gene 228 Codes Corp, Ann Arbor, MI). We then ascertained the percentage similarity of all 229 recovered sequences to available reference sequences in GenBank and BOLD, with the 230 exception of the trnH-psbA region which was not searchable within BOLD. 
232 resolution at different taxonomic depths in different taxa. For example, $r b c \mathrm{~L}$ typically 233 provides generic level resolution (CBOL plant working group, 2009) (occasionally to 234 species level), whereas the $m a t \mathrm{~K}$ and $t r n \mathrm{H}-p s b \mathrm{~A}$ regions can provide resolution to species 235 in $\sim 60-90 \%$ of cases (depending on the taxa and geographic scope) (Braukmann, 236 Kuzmina, Sills, Zakharov, \& Hebert, 2017; Burgess et al., 2011; Lahaye et al., 2008).

237 Due to incompleteness of the reference sequence databases for the flora, many sequences 238 did not show $100 \%$ identity to any species in the reference database. We therefore 239 assigned sequences to family, genus, or species depending on the region and percent 240 identity using the following criteria. For $r b c \mathrm{~L}$, sequence matches with $99.75-100 \%$ 241 identity were assigned to a genus, while matches with $99-99.75 \%$ identity were only 242 placed to a family. For $m a t \mathrm{~K}$, matches with $100 \%$ identity were assigned to a species or a 243 species cluster when more than one species in the reference set matched with $100 \%$ 244 identity; matches with 99.0-99.9\% identity were assigned to genus, while matches with $24598-99 \%$ identity were only assigned to a family. For the $t r n \mathrm{H}-p s b \mathrm{~A}$ region, most matched 246 sequences ranged from 98-99\% identity (no 100\% matches were observed). The variable 247 length of the region, the presence of repeated sequence motifs, and the small number of 248 reference sequences complicated the interpretation of BLAST analysis with the GenBank 249 dataset so most assignments were only made to a genus. For two genera, however, the $250 t r n \mathrm{H}-p s b \mathrm{~A}$ data corroborated the mat $\mathrm{K}$ designation and enabled an increased level of 251 resolution. Unique sequences for these samples were therefore designated with a number 252 (in addition to genus) and treated as putative species. Species-level designation was also 
253 accepted for sequences that matched a monotypic genus, and where sequences matched a

254 genus of three species, two of which occur outside the study area.

255

256 Method three: Metabarcoding of mixed unknowns

257 When the material to be analysed is a mixed sample of unknown taxa (in this case

258 arthropods), the entire assemblage must be targeted, followed by the use of

259 bioinformatics tools to process the sequences and ascertain the number of taxa in each

260 sample. In this case, we used DNA metabarcoding that targeted two segments of the COI

261 DNA barcode region and processed these data using a series of bioinformatics tools in a

262 well established analytical pipeline (e.g. Alberdi, Aizpurua, Gilbert, \& Bohmann, 2018;

263 Clare, Symondson, \& Fenton, 2014; Salinas-Ramos, Herrera Montalvo, León-Regagnon,

264 Arrizabalaga-Escudero, \& Clare, 2015). In brief, DNA was extracted using Qiagen Stool

265 mini-kits (Qiagen CA) with modifications from (Clare et al., 2014; Zeale, Butlin,

266 Barker, Lees, \& Jones, 2011) and eluted in 35 $\mathrm{L}$ of molecular grade water. We

267 targeted $157 \mathrm{bp}$ and $407 \mathrm{bp}$ amplicons of the DNA barcode region. PCRs were

268 conducted in $20 \mu \mathrm{l}$ reactions containing $10 \mu \mathrm{L}$ of Qiagen multiplex master mix

269 (Qiagen $\mathrm{CA}$ ), $6 \mu \mathrm{L}$ of water, $1 \mu \mathrm{L}$ of each $10 \mu \mathrm{M}$ primer and $2 \mu \mathrm{l}$ of DNA. PCR reactions

270 were: $95^{\circ} \mathrm{C}-15 \mathrm{~min} ; 50$ cycles of $95^{\circ} \mathrm{C}-30 \mathrm{sec}, 52^{\circ} \mathrm{C}-30 \mathrm{sec}, 72^{\circ} \mathrm{C}-30 \sec (1 \mathrm{~min}$

271 for the $407 \mathrm{bp}$ region); $72^{\circ} \mathrm{C}-10 \mathrm{~min}$. Amplicons were visualized on 96 well $2 \%$

272 agarose pre-cast E-gels (Invitrogen, Life Technologies). For the 157bp region we

273 used the Zeale primers (Zeale et al., 2011) which do not amplify bat DNA well,

274 modified with the two adaptor molecular identification tags (MIDs) system to

275 identify individual samples (Clare et al., 2014) without pooling. For the 407bp 
276 region we used primers MLepF1 (GCTTTCCCACGAATAAATAATA) and 277 RonMWASPdeg (GGWTCWCCWGATATAKCWTTTCC) combined in equal quantifies 278 with LepR1 (TAAACTTCTGGATGTCCAAAAATCA) and HCO2198 279 (TAAACTTCAGGGTGACCAAAAAATCA). Sequence recovery is predicted to be lower 280 with longer amplicons due to DNA degradation in digested material but longer reads 281 maximize identification. For this region we extracted and PCR-amplified all samples 282 independently but unlike the Zeal region we did not use (MIDs). This does not 283 impact MOTU estimates, but we cannot assign individual sequences to their source 284 bat so they were analysed as a pool and we do not generate networks from these 285 data, just compare MOTU estimation from alternative regions.

286 PCR products were pooled without normalization and $70 \mu \mathrm{L}$ of the pooled product 287 was cleaned using the PCRClean DX kit (Aline Biosciences) for a double size selection 288 purification protocol (Table S1). Purified PCR products were eluted in $36 \mu \mathrm{L}$ of water. 289 The concentration was measured on the Qubit 2.0 spectrophotometer using a Qubit 290 dsDNA HS Assay Kit (Invitrogen, Life Technologies). All products were normalized to $2911 \mathrm{ng} / \mu \mathrm{L}$ prior to final library dilution. Sequencing was performed using the Ion PGM 292 Template OT2 400 kit for template preparation according to manufacturer's instructions, 293 except for a $\sim 2-3 \mathrm{x}$ recommended dilution with water (Table S2) and a 316 chip. After the 294 chip check (prior to loading), the chip was flushed once with $100 \mu \mathrm{L}$ of $100 \%$ isopropanol 295 and three times with $100 \mu \mathrm{L}$ of annealing buffer.

297 Bioinformatics analysis 
The sequences were processed using two analytical pipelines for comparison

299 First, we employed well established tools in the Galaxy platform (Afgan et al., 2016).

300 Reads from the 157bp Zeal region were separated by MID allowing 2 indels and 2

301 mismatches using the barcode splitter tool. For both the 157bp and 407bp datasets

302 primers, (MIDs for the 157bp region) and adaptors were removed using the clip tool

303 (both tools from the FASTX tool kit (Assaf Gordon (2010). FASTQ/A short-reads pre-

304 processing tools. http://hannonlab.cshl.edu/fastx_toolkit/.). The resulting

305 amplicons were filtered for length $(157 \mathrm{bp}$ or $407 \mathrm{bp} \pm 10 \mathrm{bp}$ ) and dereplicated

306 (Figure S3) using the Collapose tool (FASTX tool kit). We used custom scripts to

307 remove singletons (Table S3).

308 For the 157bp dataset we clustered the remaining haplotypes into MOTUs at 309 90-97\% similarity in QIIME using the pick_otu and uclust methods 310 (http://qiime.sourceforge.net/). See Clare et al., (2016) and Flynn et al., (2015) for a

311 discussion of MOTU thresholds. For each dataset we used a BLAST analysis 312 interpreted in MEGAN (Huson, Mitra, Ruscheweyh, Weber, \& Schuster, 2011) to 313 filter out MOTUs that could not be reliably assigned to an arthropod order using a 314 reference database of $>600,000$ COI sequences extracted from GenBank. Parameters 315 in Megan were: Max Expected =0.01, Top Percent =10, Min Support Percent (0ff), 316 Min Support $=1$, Min Complexity $=0.2$. Min Score $=250$. We tested a representative 317 sequence from each MOTU in UCHIME as implemented in MOTHUR (Schloss et al., 318 2009) to filter out MOTUs that were likely to be chimeras.

319 For each MOTU dataset $(90-97 \%$ clustering) we examined a BLAST 320 assignment for MOTU representatives in MEGAN. If two or more reads were 
321 assigned to the same species we considered MOTUs oversplit, rejected that

322 threshold and tested the next most conservative option. We particularly considered

323 assignments in the Lepidoptera because this order was heavily represented in the

324 reference database. A QIIME threshold of 92 minimized MOTU oversplitting and this

325 data set was used for network construction. The same analysis was performed for

326 the 407bp dataset (MEGAN Min Score $=500$ ) but without network construction

327 (Figure S4).

328 We further queried the 157bp and 407bp datasets by comparing all sequences

329 to the same reference sequence library and to a reference library provided by D. Janzen

330 and W. Hallwachs generated from specimens (primarily Lepioptera) from the study area

331 visualised in MEGAN (Figure S5 and S6) and with custom BLAST parsing scripts. This

332 analysis extracts species-level identifications, but is biased towards identification of

333 Lepidoptera, which dominate the reference database from the study location, and the

334 accuracy of database curation (e.g., databases generally provide better resolution of

335 larger, more charismatic, and economically important species).

336 For a second comparative analytical approach we used a non-MOTU based

337 method. Initial steps were similar with reads processed in Galaxy to split by MID and

338 remove primers using cutadapt (https://cutadapt.readthedocs.io/en/stable/guide.html).

339 FastQ files were then transferred to the mBRAVE platform (http://www.mbrave.net/) and

340 processed using the parameters trim front 0bp, trim end 0bp (primers and adaptors had

341 already been removed via cutadapt) trim length 500bp and filtering of MinQV 0qv, min

342 length $147 \mathrm{bp}$, and max bases with low or ultra low QV of 100\% (to avoid specific quality

343 filter parameters. We set a pre-clustering threshold of none, and ID distance threshold of 
$3442 \%$ and left OTU thresholds as pre-set standards as we ignored MOTU analysis for this

345 comparison. Chimera screening and dereplication was performed automatically by the

346 mBRAVE platform. The resulting data is automatically compared to the BOLD system

347 library for insects using the BIN approach to attempt to associate the reads with known

348 BINs (this library contained 580, 824 reference sequences from 434, 878 known BINs,

349 last updated 21, Oct. 2018). The resulting dataset was then converted to a matrix of bat 350 species vs. associated prey BINs for further analysis.

352 Network analysis:

353 Using the data produced by all three approaches, we constructed a "network of

354 networks" in Cheddar (Hudson et al., 2013) and Bipartite (Dormann et al., 2009) in R (R

355 Development Core Team 2008) which represents all identified taxa or MOTUs. This

356 network has differing levels of resolution based on the trophic level or taxonomic group.

357 As the bats are, with a single exception, resolved to species level, they are fully

358 quantified. The mites and flies that feed on them are identified by a Barcode

359 Identification Number (BIN) (Ratnasingham \& Hebert, 2013) as a proxy for species. This

360 trophic level is also well resolved, but individual taxa are only partially quantified from

361 each bat (finding all individual parasites is not practically possible). Similarly, the plant

362 and arthropod prey levels are frequency-based as it is not possible to assess ingested plant

363 biomass from seeds (plant ID) and metabarcoding data are poorly suited to quantify the

364 biomass or abundance of species represented in the data (Deagle et al., 2018). The

365 arthropods are represented by MOTUs (Floyd et al., 2002). In addition we produced a

366 separate network of bats and prey employing the non-OTU based BIN association matrix. 


\section{Results}

369 Molecular Analysis

370 We recovered DNA barcodes from 698 bats representing 24 species belonging to

37117 genera. The barcode results generally confirmed the field IDs, but could not

372 distinguish Artibeus lituratus and Artibeus intermedius (Clare, Lim, Fenton, \& Hebert,

373 2011) leaving this node unresolved. However, other cases of taxonomic uncertainty were

374 resolved. An unknown species of Carollia was identified as C. sowelli and members of

375 two genera (Glossophaga, Micronycteris) gained species assignments. We suspect one

376 genetically divergent specimen of Sturnira parvidens may be a sister taxon, but since this

377 outcome could not be confirmed, it was retained as a single node (Figure S1).

378 We recovered DNA barcodes from 445 of the 466 mites and flies found on 18

379 host species. Parasite diversity varied from a single ectoparasite species per bat species (9

380 cases) to nine ectoparasite species on A. jamaicensis. Among the seven bat families, the

381 Emballonuridae, Mormoopidae and Phyllostomidae hosted the greatest diversity of

382 parasite species whereas individual of the Noctilionidae were only associated with two

383 ectoparasite species and individual Molossidae, Natalidae and Vespertilionidae were only

384 parasitized by one ectoparasite species at a time. Two thirds of the 34 ectoparasite

385 species, nine mites and 13 flies were only collected from one host species. The maximum

386 number of host species inhabited by a mite or fly species was four.

387 We recovered plant DNA from 112 guano samples from 12 species of bat. Guano

388 from seven bats contained two seed morphotypes analysed separately, producing 119

389 sequenced seed samples. We recovered $r b c \mathrm{~L}$ from 102 , matK from 81 and $t r n \mathrm{H}-p s b \mathrm{~A}$ 
390 from 106 samples. Through comparison to GenBank and BOLD, 103 samples had

391 sequences assigned to eight genera based on at least two of the three loci. Of these, 97

392 seed samples had sequences assigned to a putative species and 16 samples had sequences

393 placed to a genus based on a single gene region (Table $\mathrm{S} 4$ ). Comparison of $r b c \mathrm{~L}$

394 sequences to GenBank often returned multiple BLAST hits with equivalent best scores.

395 For example, top BLAST matches to Ficus or Solanum matched (100\% or 99\% identity

396 respectively) multiple species within these genera. Although some sequences did not

397 have an identical match on GenBank, all $r b c \mathrm{~L}$ sequences matched with $100 \%$ identity to a

398 sequence on BOLD, presumably reflecting the greater diversity of taxa present in the

399 latter database. Similarly, all matK sequences matched with $100 \%$ identity to sequences

400 on BOLD versus lower values on GenBank (94-100\%). In some cases this allowed a

401 more precise taxonomic assignment on BOLD, either to a species (e.g., Guazuma

402 ulmifolia), or species cluster (e.g., Cecropia obtusifolia / peltata / insignis) versus

403 assignment to a higher taxonomic rank (e.g., Urticaceae or Cecropia sp.; Ficus sp.).

404 The GenBank BLAST of $t r n \mathrm{H}-p s b \mathrm{~A}$ sequences corroborated results obtained with

$405 \quad r b c \mathrm{~L}$ and $m a t \mathrm{~K}$. In all cases, samples that yielded unique sequences for mat $\mathrm{K}$ also had

406 unique sequences for trnH-psbA. Although the limited taxonomic coverage for the latter

407 gene region on GenBank often prevented an assignment to a known species, these

408 sequence variants were treated as putative species. We also detected a probable case of

409 taxonomic error in GenBank. Two trnH-psbA sequences from our samples showed high

410 similarity (98\% identity) to Cecropia obtusifolia, an unexpected result as several other

411 sequences of almost twice the length showed nearly $100 \%$ similarity to several other 
412 species of Cecropia on GenBank. Further investigation revealed that these sequences

413 likely belong to a species of Vismia (Hypericaceae).

414 We used HTS to recover two regions of the mitochondrial COI gene (157bp, and

415 407bp, Tables S2-S3). The 157bp region has been used extensively (Alberdi et al., 2018;

416 Zeale et al., 2011), and generated high recovery rates in the present study; it is fully

417 analysed and generated 686 MOTU at the given parameters. Surprisingly, given the 418 degradation induced by digestion, the 407bp region also showed high sequencing success.

419 These two regions (Tables S5 and S6) identified a similar number of species (118 versus

420109 taxa for the $157 \mathrm{bp}$ and $407 \mathrm{bp}$ regions respectively) from all the same classes and

421 orders of arthropods (excepting one mantid). Many of the same species, for example, 32

422 species of Lepidoptera, were common in the two lists. However, there were also different

423 species identified and in a number of cases identifications were improved using the

424 longer target region. For example, sequences assigned to the genus Culex by the short

425 region could be identified as Culex nigripalpus by the $407 \mathrm{bp}$ region. This outcome

426 suggests these two regions may be complementary, adding confidence to the general

427 diversity recovered and the specific taxa identified. However, the 407bp region pushes

428 the current limits of amplicon size recovery on most HTS platforms, creating constraints

429 on quality and recovery rate. Analysis with the BIN association method in mBRAVE 430 identified 212 potential prey in the $157 \mathrm{bp}$ dataset.

432 The impact of OTUs on network metrics

433 The most novel data type generated is the metabarcoded data that underlie the bat-

434 prey network because the prey nodes do not represent a particular taxonomic level or 
435 taxon but a measure of prey genetic diversity. As a result, we investigated the impact of

436 the key bioinformatics step - that of generating MOTUs - on the measurement of

437 common network variables. Our data suggests that MOTU thresholds have a significant

438 impact on standard network metrics as taxa are lumped or split to a greater or lesser

439 extent. For most network metrics (Figure 4), an increase in the MOTU threshold (e.g.,

440 from $90 \%$ to $99 \%$ ) split taxa so the resource level in our network increased in richness

441 relative to the consumers with expected outcomes for each metric. In the case of links

442 between species, connectance, nestedness, and vulnerability this variation can result in

443 different relative rankings of these metrics between network types. For a complete

444 analysis see Hemprich-Bennett et al., (2018). The effect is consistent but less predictable

445 in measures of robustness (Figure 4), but in all cases we would have drawn the same

446 conclusion. The BIN association network (Figure 5) contained substantially fewer prey

447 nodes, which is to be expected, as the reference database for the area is minimal. Of

448 these, $75 \%$ were Lepidoptera reflecting the substantial effort to create a Lepidoptera

449 reference library for the site (see below). Interestingly, the actual measurements of

450 network properties did not differ substantially (Table 1) which reflects the tremendous

451 prey diversity represented by any method.

452

453 Discussion

454 We have demonstrated that three types of molecular data can be incorporated into

455 network analysis. DNA can be used to confirm field identities (e.g. bats) or differentiate

456 cryptic taxa (e.g. parasites) and to identify morphologically compromised material (e.g.

457 plant pulp). DNA can also be used to generate complex and fundamentally novel data via 
458 metabarcoding of mixed material (e.g. faeces) that can be analysed using MOTU or

459 association with taxa in reference collections (e.g. the BIN method). While these data

460 types can effectively generate rapid, scalable analyses of entire communities, there are

461 challenges in both generating data and in the interpretation of network metrics to ensure

462 biologically meaningful results (Table 2, Figure 4).

463 The incorporation of DNA analysis into networks presents both straightforward 464 use cases and challenges. Confirming field IDs is a common molecular procedure 465 (Borisenko, Lim, Ivanova, Hanner, \& Hebert, 2008) and differentiating cryptic or 466 taxonomically complex species is now routine (Smith, Woodley, Janzen, Hallwachs, \& 467 Hebert, 2006). These approaches have successfully been incorporated into network 468 analysis (e.g., Wirta et al., 2014). However, the inclusion of metabarcoding results is 469 more challenging and requires special consideration to integrate with network analysis.

470 Metabarcoding is best applied to mixed faecal samples, gut contents (particularly liquid 471 feeders e.g. Piñol, San Andrés, Clare, Mir, \& Symondson, 2014) or pollen carried by 472 generalists. However, it is challenging to generate reliable metabarcoded data (Alberdi et 473 al., 2018; Arrizabalaga-Escudero et al., 2018; Atwell et al., 2010; King, Read, Traugott, 474 \& Symondson, 2008). The methods of interpreting individual dietary analyses using these 475 data have been studied in several contexts (Clare et al., 2016; Flynn et al., 2015; 476 Pompanon et al., 2012; Symondson, 2002). However, certain challenges are unique to the 477 interpretation of food webs. Debate about the quantification of metabarcoding data 478 centres largely around whether sequence recovery is linked to original biomass (Deagle et 479 al., 2013; Nielsen et al., 2018; Pompanon et al., 2012). While this is possible in restricted 480 scenarios (Bowles, Schulte, Tollit, Deagle, \& Trites, 2011; Thomas et al., 2016), in many 
481 cases frequency-based measures of interactions are more appropriate (Nielsen et al., 482 2018). Frequency-based approaches are common in network ecology, for example, 483 visitation frequency to specific flowers is a standard metric of the strength of mutualistic 484 interactions (Memmott, Waser, \& Price, 2004; Vázquez, Morris, \& Jordano, 2005). 485 However, incomplete quantification needs to be considered when weighted metrics are 486 used (Kaiser-Bunbury, Muff, Memmott, Müller, \& Caflisch, 2010), as rare and common 487 interactions may be equally weighted (Clare, 2014)

488 We suggest two alternative ways to incorporate metabarcoding data: using 489 MOTUs and screening for taxonomic identities (e.g. BINs, similarity searches). The 490 advantage of MOTUs is that all data are incorporated, both known and unknown taxa. 491 However, by incorporating unknowns, one may inadvertently include non-target taxa 492 (e.g. intestinal parasites or bacteria that are not screened out bioinformatically) that may 493 generate nodes in networks unrelated to the behaviour under study or even false nodes 494 from sequencing error (Clare et al., 2016; Flynn et al., 2015). In many systems, MOTUs 495 collapse all prey levels into one "resource" level rather than revealing the complexity 496 among trophic levels. For example, in our case some insects were primary consumers 497 while others were predators, but all were treated as MOTU "prey" of an undifferentiated 498 consumer level. Our data further suggest that the protocols used to differentiate MOTUs 499 will themselves impact network metrics (Figure 4). The effect of node resolution has 500 been discussed for decades with analyses showing that the impact of resolution on node, 501 chain length and trophic levels significantly alters the observations of network properties 502 (Brose, Ostling, Harrison, \& Martinez, 2004). The situation is similar but not identical to 503 the node resolution issue of employing MOTU. The impacts of the informatic steps used 
504 to generate MOTU are only starting to be considered in ecological (Clare et al., 2016) or

505 network analysis (Hemprich-Bennett et al., 2018). Any network that incorporates taxa

506 with different levels of resolution (e.g. mixing genus and species designations) faces the

507 same challenge (Hemprich-Bennett et al., 2018; Martinez, 1991). However MOTUs make

508 it possible to easily re-analyse any dataset to empirically estimate that impact (Figure 4)

509 and one potential advantage is that MOTUs generate a uniform level of resolution in a

510 network. By their nature MOTUs represent equal and repeatable measures of biodiversity

511 (Floyd et al., 2002), even if that level does not equate to a standard level of taxonomy.

512 This may represent a powerful advantage in comparing network structure across systems,

513 but presents a challenge in interpretation. For example unnamed MOTUs of unknown

514 life-cycle and unknown affinity to each other provide limited information on the nature of

515 the ecological interaction being measured beyond the general structure of the community.

516 Similarly, if the numbers of nodes and their connections vary with analysis parameters

517 (e.g. MOTU threshold) a network on its own holds little biological meaning. However, if

518 the same methods are replicated a biological picture can emerge. For example, if the prey

519 level undergoes a population crash, the genetic diversity and the MOTU number would

520 similarly decline relative to the consumer level and fluctuations in parameters such as

521 generality or nestedness would be measureable. The key then is to compare only analyses

522 that employ the same methods from sequencing platform and field and lab protocol to

523 informatics choices, just as sampling protocols and node resolution should be maintained

524 in traditional networks being compared. This would be required to avoid context

525 specificity. It is also necessary to pick specific metrics; for example network level metrics 
526 may be more reliable than motif measurements (though see Hemprich-Bennett et al., 527 2018).

528 In contrast, similarity based searchers and BIN association type data provid better

529 ability to determine exactly what is being included as a node (e.g. Figure 5) and yield

530 greater ecological information about the type of interaction being measured, but will be

531 biased by the contents of the reference library being used. In this case, the network

532 metrics were similar enough that comparative conclusions about bat-prey/BIN, bat-

533 parasite and bat-plant networks would remain the same but some specific indicators

534 change. For example, generality of the bat-BIN network was much lower reflecting the

535 substantial reduction in prey nodes when relying exclusively on reference collections for

536 the inclusion of a prey node. As reference collections improve this effect will diminish

537 but it is a very important factor in relatively unexplored faunas.

539 Three distinct data types

540 This paper has considered three distinct types of molecular data. The bat and plant

541 identifications provide by DNA deliver nearly perfect resolution of the network. Such

542 analysis generates data similar to that employed in traditional network ecology, the only

543 major difference being the need for multi-locus data to obtain species-level resolution for

544 plants. The parasite identifications were generated in a similar fashion to the bat data (one

545 sequence per specimen), but with the crucial difference that current reference databases

546 are very incomplete. As a consequence, we employed an alternative taxonomic system,

547 the Barcode Index Number (BIN). The performance of the BIN system has been

548 extensively tested (Ratnasingham \& Hebert, 2013) and these studies have shown that it 
549 delivers taxonomic resolution that is very close to traditional taxonomy. This data type

550 (Table 2) has the advantage of making it possible to incorporate taxa which are

551 apparently different species but where the current taxonomic system is incomplete. BIN

552 analysis avoids unresolved nodes in network construction, but imposes a constraint that

553 the identification is based on a measure of sequence differentiation observed in related

554 taxa. Unlike other MOTU-generating methods, the BIN system is not based on strict $a$

555 prior threshold delimitation but has been trained specifically using the large Sanger data

556 sets for the DNA barcode region. In a test of 1400 species spanning birds, bees, fishes

557 and Lepidoptera, the correspondence between species counts based on traditional

558 taxonomy and BINs was very high $\left(\mathrm{r}^{2}=0.99\right)$ and the actual mapping of species to BIN

559 was approximately $90 \%$ (varying from $79 \%-97 \%$ between taxonomic group)

560 (Ratnasingham \& Hebert, 2013). Thus, when viewed from the context of DNA

561 barcoding, BINs are a strong proxy for species. Because the definition of new BINs

562 requires at least 500bp of sequence information from the COI barcode region, the short

563 reads generated by most current HTS platforms cannot be used to delineate new BINs

564 although they can be matched to existing BINs. Reflecting this constraint, there is a need

565 for other methods of MOTU generation. For this third data type, data are most often

566 analysed using MOTU without much (if any) taxonomic identification (e.g. Figure 1).

567 This has the advantage of making it possible to analyzed mixed sources (e.g. stomach

568 contents) but imposes unique problems for network ecology as it compresses trophic

569 levels and dispenses with traditional taxonomy. While such analysis can generate data for

570 a comparable interaction network model, it may not represent a trophic food web. In the

571 study location, most arthropods remain undescribed despite decades of intense taxonomic 
572 work. For example, some 400,000 arthropod species are estimated to be present in the

573 ACG, but just 43,000 of these species have been barcoded over 14 years, revealing the

574 scope of the taxonomic challenge (D.H. Janzen Pers. Comm.). In such locations, a

575 complete food web or interaction network is impossible and restricting analysis to those

576 species which can gain a full taxonomic designation (either by morphology or DNA)

577 would introduce a substantial bias (Table S6). As a consequence our bat-BIN network

578 contained substantially fewer nodes than our bat-prey network based on MOTU and

579 would be less comparable to a network generated in an area with a different/reduced

580 reference database. In such cases, a BIN or MOTU approach to generating a reference

581 collection and then some sort of association or matching system is the only means of

582 developing an ecosystem network model. The use of reference databases can to provide a

583 familiar binomial designation on some nodes by similarity searchers or BIN association

584 but imposes a significant bias on the data, which is then composed of "things found in

585 databases" while novel BINs, and MOTUs do not impose this bias. On the other hand,

586 novel BINs and MOTUs may include non-target taxa such as parasites, parasitoids, or

587 taxa acquired via secondary predation.

588

589 Comparison of 157 and 407bp datasets:

590 Current consensus suggests that short reads are required to maximize MOTU

591 and taxonomic ID recovery in digested material because of DNA degradation.

592 Contrary to this expectation, the 407bp region had higher MOTU estimates and

593 broader taxonomic coverage when evaluated using BLAST, suggesting it has less

594 amplification bias and hence a complementary region for arthropod diversity 
595 analysis. However, this conclusions need to be considered with caution. Longer 596 reads should generate better taxon identification scores (more information) but will 597 also generate high rates of low quality BLAST scores (local alignments). We 598 modified the MEGAN Min Support value to partially compensate for this and to 599 maximize assignment with most scores $>98 \%$ similar to references. Taxonomic 600 assignments of MOTUs (e.g. Table S5 and S6) should be considered for interest's 601 sake only in this dataset, particularly when a reference databases contains errors or 602 skewed coverage. For this reason we considered only MOTUs for network analysis. 603 Despite the promise of the $407 \mathrm{bp}$ region we used the $157 \mathrm{bp}$ region MOTUs for 604 network analysis for two reasons. First, unlike the $157 \mathrm{bp}$ region, the $407 \mathrm{bp}$ region is 605 new to NGS analysis and has not been evaluated for this purpose before. We 606 consider it an interesting and potentially important tool but are hesitant to rely on it 607 until further testing has been completed. Second, the 407bp region is long compared 608 to the capacity of most high throughput sequencing platforms which limits its use 609 and prevented us from employing MIDs to separate samples. Platform read length 610 has generally fallen since the first highly popular Roche454 platform capable of 611 1000bp reads to the now standard 250bp paired end reads of the MiSeq, thus while 612 promising, the $407 \mathrm{bp}$ read will be analytically challenging. Newer platforms such as 613 the SMRT sequencing platform (PACBIO, Pacific Biosciences) can overcome this 614 problem allowing longer reads and thus higher taxonomic resolution assuming that 615 digestion has not substantially compromised the DNA.

616

617 DNA integration into network ecology. 
619 First, the technique does not rely on the need to observe interactions or the time 620 consuming rearing practices used to establish many cases of parasitism (Wirta et al.,

621 2014). It can be applied to broken seeds, fruit pulp (e.g. Lim et al., 2017), single grains of 622 pollen or morphologically destroyed material (e.g., digested remains) as well as entire 623 specimens. Even traces of DNA (eDNA) with no observable material are amenable 624 (Bohmann et al., 2014; Drinkwater, Clare, \& Rossiter, In Review); for example, seeds 625 dropped on the forest floor will have DNA of the plant, but also of the animal that 626 dropped them from either saliva or cells from a digestive tract. González-Varo et al., 627 (2014) have spectacularly demonstrated this method to capture bird DNA on the surface 628 of dispersed seeds. Similarly, the detection of cryptic species and relationships represents 629 a huge shift in the resolution of interaction networks. This was demonstrated by Wirta et 630 al., (2014) who observed that DNA dramatically increased the number of identified 631 interaction types and altered the perceived host specificity of host-parasitoid networks.

632 A rapid DNA-based network biomonitoring tool will require us to understand: first, 633 which data can be quantified (Deagle et al., 2018) and second, which metrics are reliable, 634 in relative or absolute terms, to ensure we produce biologically meaningful outputs 635 (Hemprich-Bennett et al., 2018, Clare et al. 2016, Ings et al. 2009, ). However, these 636 datasets are already being demonstrated as powerful tools to resolve complex interaction 637 networks quickly and in exquisite detail. Here we have generated a detailed network of 638 networks in a complex tropical ecosystem incorporating different molecular data types as 639 a case study. Ecologically, our data suggest a hitherto unrecognised keystone species and 640 behavioural flexibility that may be critical to the success of insectivores (Box 1). 
641 Methodologically, our findings support the approach, but also highlight the need for

642 rigorous testing of methods. The rapidly advancing technology of this field means that

643 such analyses will soon become a common and relatively inexpensive tool for

644 understanding biostructure (McCann, 2007). While a fully resolved and taxonomically

645 identified network will always be the goal, our analysis demonstrates the utility of these

646 tools for network ecology and produces the first full network of networks resolved

647 entirely by DNA.

648

649 Acknowledgments: We are indebted to collaborators who have supported this research

650 including staff at the Canadian Centre for DNA Barcoding and the Biodiversity Institute

651 of Ontario and the staff of Sector Santa Rosa in the Area de Conservación Guanacaste

652 (ACG) Costa Rica particularly Daniel Janzen, Winnie Hallwachs, Roger Blanco and

653 Maria Marta Chavarria.

654

655

656

657

658

659

660

661

662

663

664

665

666

667

668

669

670

671

\section{References}

Afgan, E., Baker, D., van den Beek, M., Blankenberg, D., Bouvier, D., Čech, M., ... Goecks, J. (2016). The Galaxy platform for accessible, reproducible and collaborative biomedical analyses: 2016 update. Nucleic Acids Research, 44(W1), W3-W10. doi:10.1093/nar/gkw343

Alberdi, A., Aizpurua, O., Gilbert, M. T. P., \& Bohmann, K. (2018). Scrutinizing key steps for reliable metabarcoding of environmental samples. Methods in Ecology and Evolution, 9, 134-147. doi:10.1111/2041-210X.12849

Arrizabalaga-Escudero, A., Clare, E. L., Salsamendi, E., Alberdi, A., Garin, I., Aihartza, J., \& Goiti, U. (2018). Assessing niche partitioning of co-occurring sibling bat species by DNA metabarcoding. Molecular Ecology, DOI: 10.1111/mec.14508. doi:10.1111/mec.14508

Atwell, S., Huang, Y. S., Vilhjálmsson, B. J., Willems, G., Horton, M., Li, Y., ... Nordborg, M. (2010). Genome-wide association study of 107 phenotypes in Arabidopsis thaliana inbred lines. Nature, 465(7298), 627-631. doi:10.1038/nature08800 
672

673

674

675

676

677

678

679

680

681

682

683

684

685

686

687

688

689

690

691

692

693

694

695

696

697

698

699

700

701

702

703

704

705

706

707

708

709

710

711

712

Bohmann, K., Evans, A., Gilbert, M. T. P., Carvalho, G. R., Creer, S., Knapp, M., ... de Bruyn, M. (2014). Environmental DNA for wildlife biology and biodiversity monitoring. Trends in Ecology \& Evolution, 29(6), 358-367. doi:http://dx.doi.org/10.1016/j.tree.2014.04.003

Borisenko, A. V, Lim, B. K., Ivanova, N. V, Hanner, R. H., \& Hebert, P. D. N. (2008). DNA barcoding in surveys of small mammal communities: a field study in Suriname. Molecular Ecology Resources, 8(3), 471-479. doi:10.1111/j.14718286.2007.01998.x

Bowles, E., Schulte, P. M., Tollit, D. J., Deagle, B. E., \& Trites, A. W. (2011). Proportion of prey consumed can be determined from faecal DNA using real-time PCR. Molecular Ecology Resources, 11(3), 530-540. doi:10.1111/j.17550998.2010.02974.x

Braukmann, T. W. A., Kuzmina, M. L., Sills, J., Zakharov, E. V., \& Hebert, P. D. N. (2017). Testing the Efficacy of DNA Barcodes for Identifying the Vascular Plants of Canada. PLOS ONE, 12(1), e0169515. doi:10.1371/journal.pone.0169515

Brose, U., Ostling, A., Harrison, K., \& Martinez, N. D. (2004). Unified spatial scaling of species and their trophic interactions. Nature, 428(6979), 167-171. Retrieved from http://dx.doi.org/10.1038/nature02297

Burgess, K. S., Fazekas, A. J., Kesanakurti, P. R., Graham, S. W., Husband, B. C., Newmaster, S. G., ... Barrett, S. C. H. (2011). Discriminating plant species in a local temperate flora using the rbcL+matK DNA barcode. Methods in Ecology and Evolution, 2(4), 333-340. doi:10.1111/j.2041-210X.2011.00092.x

CBOL plant working group. (2009). A DNA barcode for land plants. Proceedings of the National Academy of Sciences of the United States of America, 106(31), 1279412797. doi:10.1073/pnas.0905845106

Christe, P., Arlettaz, R., \& Vogel, P. (2000). Variation in intensity of a parasitic mite (Spinturnix myoti) in relation to the reproductive cycle and immunocompetence of its bat host (Myotis myotis). Ecology Letters, 3(3), 207-212. doi:10.1046/j.14610248.2000.00142.x

Clare, E. L. (2014). Molecular detection of trophic interactions: emerging trends, distinct advantages, significant considerations and conservation applications. Evolutionary Applications, 7(9), 1144-1157.

Clare, E. L., Barber, B. R., Sweeney, B. W., Hebert, P. D. N., \& Fenton, M. B. (2011). Eating local: influences of habitat on the diet of little brown bats (Myotis lucifugus). Molecular Ecology, 20(8), 1772-1780. doi:10.1111/j.1365-294X.2011.05040.x

Clare, E. L., Chain, F. J. J., Littlefair, J. E., \& Cristescu, M. E. (2016). The effects of parameter choice on defining molecular operational taxonomic units and resulting ecological analyses of metabarcoding data. Genome, 59(11), 981-990. doi:10.1139/gen-2015-0184

Clare, E. L., Goerlitz, H. R., Drapeau, V. A., Holderied, M. W., Adams, A. M., Nagel, J., ... Brock Fenton, M. (2014). Trophic niche flexibility in Glossophaga soricina: How 
713

715

716

717

718

719

720

721

722

723

724

725

726

727

728

729

730

731

732

733

734

735

736

737

738

739

740

741

742

743

744

745

746

747

748

749

750

751

752

a nectar seeker sneaks an insect snack. Functional Ecology, 28(3).

doi:10.1111/1365-2435.12192

Clare, E. L., Lim, B. K., Fenton, M. B., \& Hebert, P. D. N. (2011). Neotropical bats: estimating species diversity with DNA barcodes. PloS ONE, 6(7), e22648. doi:10.1371/journal.pone.0022648

Clare, E. L., Symondson, W. O. C., \& Fenton, M. B. (2014). An inordinate fondness for beetles? Variation in seasonal dietary preferences of night roosting big brown bats (Eptesicus fuscus). Molecular Ecology, 23(15), 2333-2647. doi:10.1111/mec.12519

Costanza, R., D’Arge, R., de Groot, R., Farber, S., Grasso, M., Hannon, B., ... van den Belt, M. (1997). The value of the world's ecosystem services and natural capital. Nature, 387(6630), 253-260. doi:doi: 10.1038/387253a0

Deagle, B. E., Thomas, A. C., McInnes, J. C., Clarke, L. J., Vesterinen, E. J., Clare, E. L., ... Eveson, J. P. (2018). Counting with DNA in metabarcoding studies: How should we convert sequence reads to dietary data? Molecular Ecology. doi: $10.1111 / \mathrm{mec} .14734$

Deagle, B. E., Thomas, A. C., Shaffer, A. K., Trites, A. W., \& Jarman, S. N. (2013). Quantifying sequence proportions in a DNA-based diet study using Ion Torrent amplicon sequencing: which counts count? Molecular Ecology Resources, 13(4), 620-633. doi:10.1111/1755-0998.12103

Dormann, C. F., Fründ, J., Blüthgen, N., \& Gruber, B. (2009). Indices, graphs and null models: analyzing bipartite ecological networks. The Open Ecology Journal, 7-24.

Drinkwater, R., Clare, E. L., \& Rossiter, S. J. (n.d.). Applying iDNA to compare the feeding strategies of two haematophagous leech species in North Borneo. Molecular Ecology.

Evans, D. M., Kitson, J. J. N., Lunt, D. H., Straw, N. A., \& Pocock, M. J. O. (2016). Merging DNA metabarcoding and ecological network analysis to understand and build resilient terrestrial ecosystems. Functional Ecology, 30(12), 1904-1916. doi:10.1111/1365-2435.12659

Fazekas, A. J., Burgess, K. S., Kesanakurti, P. R., Graham, S. W., Newmaster, S. G., Husband, B. C., ... Barrett, S. C. H. (2008). Multiple multilocus DNA barcodes from the plastid genome discriminate plant species equally well. PloS One, 3(7), e2802. doi:10.1371/journal.pone.0002802

Floyd, R., Abebe, E., Papert, A., \& Blaxter, M. (2002). Molecular barcodes for soil nematode identification. Molecular Ecology, 11(4), 839-850.

Flynn, J. M., Brown, E. A., Chain, F. J. J., MacIsaac, H. J., \& Cristescu, M. E. (2015). Toward accurate molecular identification of species in complex environmental samples: testing the performance of sequence filtering and clustering methods. Ecology and Evolution, 5(11), 2252-2266. doi:10.1002/ece3.1497

Ford, C. S., Ayres, K. L., Toomey, N., Haider, N., Van Alphen Stahl, J., Kelly, L. J., ... Wilkinson, M. J. (2009). Selection of candidate coding DNA barcoding regions for 
use on land plants. Botanical Journal of the Linnean Society, 159(1), 1-11. doi:10.1111/j.1095-8339.2008.00938.x

Fritz, G. N. (1983). Biology and ecology of bat flies (Diptera:Streblidae) on bats in the genus Carollia. Journal of Medical Entomology, 20, 1-10.

Gibson, J. F., Shokralla, S., Curry, C., Baird, D. J., Monk, W. A., King, I., \& Hajibabaei, M. (2015). Large-scale biomonitoring of remote and threatened ecosystems via high-throughput sequencing. PLOS ONE, 10(10), e0138432. doi:10.1371/journal.pone.0138432

González-Varo, J. P., Arroyo, J. M., \& Jordano, P. (2014). Who dispersed the seeds? The use of DNA barcoding in frugivory and seed dispersal studies. Methods in Ecology and Evolution, 5(8), 806-814. doi:10.1111/2041-210X.12212

Guimarães Jr, P. R., Jordano, P., \& Thompson, J. N. (2011). Evolution and coevolution in mutualistic networks. Ecology Letters, 14(9), 877-885. doi:10.1111/j.14610248.2011.01649.x

Hebert, P. D. N., Cywinska, A., Ball, S. L., \& DeWaard, J. R. (2003). Biological identifications through DNA barcodes. Proceedings of the Royal Society BBiological Sciences, 270(1512), 313-321. doi:10.1098/rspb.2002.2218

Hebert, P. D. N., Dewaard, J. R., Zakharov, E. V, Prosser, S. W. J., Sones, J. E., McKeown, J. T., ... La Salle, J. (2013). A DNA “barcode blitz": rapid digitization and sequencing of a natural history collection. PloS One, 8(7), e68535. doi:10.1371/journal.pone.0068535

Hebert, P. D. N., Ratnasingham, S., \& DeWaard, J. R. (2003). Barcoding animal life: cytochrome $c$ oxidase subunit 1 divergences among closely related species. Proceedings of the Royal Society of London B-Biological Sciences, 270 (Suppl, S96S99.

Hemprich-Bennett, D. R., Oliveira, H. F. M., Le Comber, S. C., Rossiter, S. J., \& Clare, E. L. (2018). Assessing the impact of taxon resolution on network structure, with implication for comparative ecology. BioRxiv. Retrieved from http://biorxiv.org/content/early/2018/06/28/357376.abstract

Hudson, L. N., Emerson, R., Jenkins, G. B., Layer, K., Ledger, M. E., Pichler, D. E., ... Reuman, D. C. (2013). Cheddar: analysis and visualisation of ecological communities in R. Methods in Ecology and Evolution, 4(1), 99-104. doi:10.1111/2041-210X.12005

Huson, D. H., Mitra, S., Ruscheweyh, H.-J., Weber, N., \& Schuster, S. C. (2011). Integrative analysis of environmental sequences using MEGAN4. Genome Research, 21(9), 1552-1560. doi:10.1101/gr.120618.111

Ings, T. C., Montoya, J. M., Bascompte, J., Blüthgen, N., Brown, L., Dormann, C. F., ... Woodward, G. (2009). Review: Ecological networks - beyond food webs. Journal of Animal Ecology, 78(1), 253-269. doi:10.1111/j.1365-2656.2008.01460.x

Kaiser-Bunbury, C. N., Muff, S., Memmott, J., Müller, C. B., \& Caflisch, A. (2010). The 
793

794

795

796

797

798

799

800

801

802

803

804

805

806

807

808

809

810

811

812

813

814

815

816

817

818

819

820

821

822

823

824

825

826

827

828

829

830

831

robustness of pollination networks to the loss of species and interactions: a quantitative approach incorporating pollinator behaviour. Ecology Letters, 13(4), 442-452. doi:10.1111/j.1461-0248.2009.01437.x

King, R. A., Read, D. S., Traugott, M., \& Symondson, W. O. C. (2008). Molecular analysis of predation: a review of best practice for DNA-based approaches. Molecular Ecology, 17(4), 947-963. doi:10.1111/j.1365-294X.2007.03613.x

Kress, W. J., Wurdack, K. J., Zimmer, E. A., Weigt, L. A., \& Janzen, D. H. (2005). Use of DNA barcodes to identify flowering plants. Proceedings of the National Academy of Sciences of the United States of America, 102(23), 8369-8374.

Lahaye, R., van der Bank, M., Bogarin, D., Warner, J., Pupulin, F., Gigot, G., ... Savolainen, V. (2008). DNA barcoding the floras of biodiversity hotspots. Proceedings of the National Academy of Sciences of the United States of America, 105(8), 2923-8. doi:10.1073/pnas.0709936105

Lim, V.-C., Clare, E. L., Littlefair, J. E., Ramli, R., Bhassu, S., \& Wilson, J.-J. (2017). Impact of urbanisation and agriculture on the diet of fruit bats. Urban Ecosystems, 21(1), 61-70. doi:10.1007/s11252-017-0700-3

Martín González, A. M., Dalsgaard, B., \& Olesen, J. M. (2010). Centrality measures and the importance of generalist species in pollination networks. Ecological Complexity, 7(1), 36-43. doi:10.1016/j.ecocom.2009.03.008

Martinez, N. D. (1991). Artifacts or Attributes? Effects of Resolution on the Little Rock Lake Food Web. Ecological Monographs, 61(4), 367-392. doi:10.2307/2937047

McCann, K. (2000). The diversity-stability debate. Nature, 405(6783), 228-233. doi: $10.1038 / 35012234$

McCann, K. (2007). Protecting biostructure. Nature, 446(7131), 29. doi:10.1038/446029a

McDonald-Madden, E., Sabbadin, R., Game, E. T., Baxter, P. W. J., Chadès, I., \& Possingham, H. P. (2016). Using food-web theory to conserve ecosystems. Nature Communications, 7, 10245. Retrieved from http://dx.doi.org/10.1038/ncomms10245

Memmott, J., Waser, N., \& Price, M. (2004). Tolerance of pollination networks to species extinctions. Proceedings of the Royal Society of London B-Biological Sciences, 271(1557), 2605-2611.

Milo, R., Shen-Orr, S., Itzkovitz, S., Kashtan, N., Chklovskii, D., \& Alon, U. (2002). Network Motifs: Simple building blocks of complex networks. Science, 298(5594), 824-827.

Nielsen, J. M., Clare, E. L., Hayden, B., Brett, M. T., \& Kratina, P. (2018). Diet tracing in ecology: method comparison and selection. Methods in Ecology and Evolution, 9(2), 278-291. doi:10.1111/2041-210X.12869

Nuismer, S. L., Jordano, P., \& Bascompte, J. (2013). Coevolution and the architecture of mutualistic networks: coevolving networks. Evolution, 67(2), 338-354.

doi:10.1111/j.1558-5646.2012.01801.x 
832

833

834

835

836

837

838

839

840

841

842

843

844

845

846

847

848

849

850

851

852

853

854

855

856

857

858

859

860

861

862

863

864

865

866

867

868

869

870

871

Piñol, J., San Andrés, V., Clare, E. L., Mir, G., \& Symondson, W. O. C. (2014). A pragmatic approach to the analysis of diets of generalist predators: the use of nextgeneration sequencing with no blocking probes. Molecular Ecology Resources, 14(1), 18-26. doi:10.1111/1755-0998.12156

Pocock, M. M. J. O., Evans, D. M. D., \& Memmott, J. (2012). The robustness and restoration of a network of ecological networks. Science, 335(6071), 973-977.

Poisot, T., Stouffer, D. B., \& Kéfi, S. (2016). Describe, understand and predict: why do we need networks in ecology? Functional Ecology, 30, 1878-1882. doi:10.1111/1365-2435.12799

Pompanon, F., Deagle, B. E., Symondson, W. O. C., Brown, D. S., Jarman, S. N., \& Taberlet, P. (2012). Who is eating what: diet assessment using next generation sequencing. Molecular Ecology, 21(8), 1931-1950. doi:10.1111/j.1365294X.2011.05403.x

Porco, D., Rougerie, R., Deharveng, L., \& Hebert, P. D. N. (2010). Coupling nondestructive DNA extraction and voucher retrieval for small soft-bodied Arthropods in a high-throughput context: the example of Collembola. Molecular Ecology Resources, 10(6), 942-945. doi:10.1111/j.1755-0998.2010.2839.x

R Development Core Team: R: A language and environment for statistical computing. (2008). Retrieved from http://www.r-project.org

Ratnasingham, S., \& Hebert, P. D. N. (2007). BOLD: the barcode of life data system (www.barcodinglife.org). Molecular Ecology Notes, 7(3), 355-364. doi:10.1111/j.1471-8286.2007.01678.x

Ratnasingham, S., \& Hebert, P. D. N. (2013). A DNA-based registry for all animal species: the Barcode Index Number (BIN) system. PloS One, 8(7), e66213. doi:10.1371/journal.pone.0066213

Reid, F. A. (2009). A field guide to the mammals of central america and southeast mexico. Oxford University Press.

Roslin, T., \& Majaneva, S. (2016). The use of DNA barcodes in food web construction — terrestrial and aquatic ecologists unite! Genome, 59(9), 603-628.

Salinas-Ramos, V. B., Herrera Montalvo, L. G. G., León-Regagnon, V., ArrizabalagaEscudero, A., \& Clare, E. L. (2015). Dietary overlap and seasonality in three species of mormoopid bats from a tropical dry forest. Molecular Ecology, 24(20), 52965307. doi:10.1111/mec. 13386

Schloss, P., Westcott, S. L., Ryabin, T., Harrmann, M., Hollister, E. B., Lesniewski, R. A., ... Weber, C. F. (2009). Introducing mothur: Open-source, platformindependent, community-supported software for describing and comparing microbial communities. Applied and Environmental Microbiology, 75(23), $7537-$ 7541.

Simmons, N. B. (2005). Order Chiroptera. In D. E. Wilson \& D. M. Reeder (Eds.), Mammal Species of the world: a taxonomic and geographic reference, Volume 1 
872

873

874

875

876

877

878

879

880

881

882

883

884

885

886

887

888

889

890

891

892

893

894

895

896

897

898

899

900

901

902

903

904

905

906

907

908

909 (3rd editio, pp. 312-529). Johns Hopkins Univeristy Press.

Smith, M. A., Woodley, N. E., Janzen, D. H., Hallwachs, W., \& Hebert, P. D. N. (2006). DNA barcodes reveal cryptic host-specificity within the presumed polyphagous members of a genus of parasitoid flies (Diptera: Tachinidae). Proceedings of the National Academy of Sciences of the United States of America, 103(10), 3657-3662. doi:10.1073/pnas.0511318103

Strona, G., \& Lafferty, K. D. (2016). Environmental change makes robust ecological networks fragile. Nature Communications, 7, 12462. Retrieved from http://dx.doi.org/10.1038/ncomms 12462

Symondson, W. O. C. (2002). Molecular identification of prey in predator diets. Molecular Ecology, 11(4), 627-641.

Thébault, E., \& Fontaine, C. (2010). Stability of ecological communities and the architecture of mutualistic and trophic networks. Science, 329(5993), 853-856. Retrieved from http://science.sciencemag.org/content/329/5993/853.abstract

Thomas, A. C., Deagle, B. E., Eveson, J. P., Harsch, C. H., \& Trites, A. W. (2016). Quantitative DNA metabarcoding: improved estimates of species proportional biomass using correction factors derived from control material. Molecular Ecology Resources, 16(3), 714-726. doi:10.1111/1755-0998.12490

Toju, H. (2015). High-throughput DNA barcoding for ecological network studies. Population Ecology, 57(1), 37-51. doi:10.1007/s10144-014-0472-z

Vázquez, D. P., Morris, W. F., \& Jordano, P. (2005). Interaction frequency as a surrogate for the total effect of animal mutualists on plants. Ecology Letters, 8(10), 10881094. doi:10.1111/j.1461-0248.2005.00810.x

Wirta, H. K., Hebert, P. D. N., Kaartinen, R., Prosser, S. W., Várkonyi, G., \& Roslin, T. (2014). Complementary molecular information changes our perception of food web structure. Proceedings of the National Academy of Sciences of the United States of America, 111(5), 1885-1890. doi:10.1073/pnas.1316990111

Worm, B., Barbier, E. B., Beaumont, N., Duffy, J. E., Folke, C., Halpern, B. S., ... Watson, R. (2006). Impacts of biodiversity loss on ocean ecosystem services. Science, 314(5800), 787-790. Retrieved from http://science.sciencemag.org/content/314/5800/787.abstract

Zeale, M. R. K., Butlin, R. K., Barker, G. L. A., Lees, D. C., \& Jones, G. (2011). Taxonspecific PCR for DNA barcoding arthropod prey in bat faeces. Molecular Ecology Resources, 11(2), 236-244. doi:10.1111/j.1755-0998.2010.02920.x 
Field Methods: All materials were acquired from past research at the field location and/or held in personal collections. All bats were caught over a six-week period from late May - early July of 2009 using mistnets or harptraps in Sector Santa Rosa of the Area de Conservación Guanacaste. Net locations were alternated nightly between the Bosque Humeda, La Casona and the Picnic area with an extra netting night at the Playa Naranjo targeting Noctilio. Each bat was identified and placed in a cloth bag for approximately one hour. Any guano produced was collected for taxonomic identification of prey items and the bats were released at the point of capture. Ectoparasites and wing biopsies were preserved in isopropyl alcohol; guano was frozen. Morphological identification of the ectoparasites to fly or mite was made in order to separte functional groups. Guano samples were screened for seed fragments and insect remains and classified as containing plant material or insect material. Two genera, Glossophaga and Micronycteris, were left with provisional species level ID. Artibeus lituratus and A. intermedius, could not be distinguished in the field and are referred to as $A$. $s p$.

Network Analysis: We visualised the interaction networks using Bipartite (Dormann et al., 2009) and Cheddar (Hudson et al., 2013) as implemented in R (R Development Core Team, 2015). We compared the structural metrics (links per species, asymmetry, connectance, nestedness, generality, and vulnerability) of each traditional bipartite sub-network (bat-parasite, bat-plant, bat-insect). We evaluated the robustness of each network and modelled the effects of species loss and restoration within the networks. We employed three extinction models: species removed randomly (null model), species removed from most to least connected (Rdworst case scenario) (Kaiser-Bunbury, Muff, Memmott, Müller, \& Caflisch, 2010) and species removed from least to most frequently detected (Ra-best case scenario). Species lose connections within the network when their hosts, prey, predators, or mutualists are eliminated. From each of our three component networks (parasitism, mutualism, predation) we measured network robustness (Kaiser-Bunbury et al., 2010; Memmott, Waser, \& Price, 2004). We then modelled a restoration scenario where bat species are re-introduced from greatest to least connected (best-case) and assessed the proportion of links restored to the structure. To pinpoint possible keystone species, we examined the role of each bat species within the entire network of network using betweenness and closeness centrality scores (Martín González, Dalsgaard, \& Olesen, 2010) in igraph (Csardi and Nepusz 2006). For simplicity, when individual networks are depicted, we present bats on the top rather than arranging these by trophic level (bats occupy multiple trophic levels making any other display exceedingly complex). Finally, we analysed the impact of OTU clustering thresholds of insects in the bat-prey network on the measurement of these metrics considering clustering thresholds from $90-99 \%$.

A network of networks: Using these data, we present the first "network of networks" where all underlying data have been generated using a molecular approach (Figure 1a). We evaluated the structural metrics (Table 1) and robustness (Figure 2) of 
traditional sub-networks (Figure 1bcd) and modelled the effects of bat species loss on parasite persistence (Figure 2a), plant mutualism (Figure 2b), predation (Figure $2 \mathrm{c}$ ), and secondary extinction of bats from prey loss (Figure 2d). Under all models, parasite networks were less robust $(\mathrm{Ra}=0.69 / \mathrm{Rd}=0.36)$ and mutualistic relationships were only slightly more robust $(R a=0.74 / R d=0.4)$. However, arthropods responded differently to models of extinction: a high proportion of prey face predation, even when the highest-ranking bat species by abundance are eliminated $(\mathrm{Ra}=0.86)$, while, conversely, arthropods experience a tremendous release from predation when bat species go extinct by connectance $(\mathrm{Rd}=0.28)$. Insectivorous bats appear robust to the loss of prey species $(\mathrm{Ra}=0.998 / \mathrm{Rd}=0.85)$. Even under the worst-case scenario, the first bat species is not lost until $32 \%$ of arthropods are extinct, and even when $>90 \%$ of arthropods are lost, $>70 \%$ of bat species remain in the network if prey biomass was sufficient (Figure 2d). Only G. soricina showed significant trophic flexibility operating in both a mutualistic and strong predatory role (high centrality scores, Supplemental Information Table S8). This is also evident in our restoration ecology model (Figure 3) where the third bat returned based on connectance is G. soricina introducing parasites, insects, and plants at the same time.

A snap shot of a bat community: Even considering the variability of metrics across multiple MOTU resolutions (Figure 4), the generality of bat-prey networks is extreme compared to the bat-parasite and bat-plant networks. This significantly impacts on our understanding of robustness in this system and may provide evidence in the diversity vs. stability debate (McCann, 2000). The data suggest extraordinary behavioural flexibility of insectivorous bats and their lack of reliance on specific prey. While there is evidence for resource specialisation (e.g. the preference for beetles in Eptesicus (Clare, Symondson, \& Fenton, 2014) or moths in sibling rhinolophids (Arrizabalaga-Escudero et al., 2018)) most studies that have employed molecular techniques have observed very generalist flexible behaviour in foraging (Salinas-Ramos, Herrera Montalvo, León-Regagnon, Arrizabalaga-Escudero, \& Clare, 2015; Sedlock, Krüger, \& Clare, 2014) though none have examined a community on this scale. Second, perhaps the most interesting observation is the position of the bat Glossophaga soricina in the network. Clare, Goerlitz, et al., (2013) used a molecular dietary analysis to identify a novel hunting strategy that permits this supposed "nectar bat" to sneak up on insects. The bats' echolocation calls are low enough in intensity that prey with ears do not detect the approaching threat in time to evade it (Clare, Goerlitz, et al., 2013). Our network analysis suggests that insectivory in Glossophaga is not a rare behaviour but rather, during the period of this study, G. soricina was the third best-connected insectivore in the community in addition to its role in pollination and, seed dispersal and as a parasite host. Its diverse functional roles make it a probable keystone species with very high betweeness and closeness centrality (Supplemental Information Table S8). This distinguishes it as the only bat occupying all these functional roles in the network and thus a species of special conservation interest. In contrast some species are only very tangentially associated with this network. For example Desmodus rotundus, the 
1001 vampire bat, is connected to only one parasite and thus forms its own module of 1002 interactions unconnected to the rest of the community.

1005 Data Accessibility: All molecular data can be found in Dryad 1006 https://doi.org/10.5061/dryad.0k90c0v and BOLD projects (BCCR Bats of Costa Rica 1007 ACG \& BCPB Parasites of tropical bats) also contain sequences and collection metadata 1008 and associated GenBank accessions.

1011 Author Contributions. ELC, AMA, and JN performed fieldwork. ELC, AJF, NVI and 1012 RMF performed laboratory work. ELC and RG performed analysis. PDNH, MBF and 1013 SGN helped design the study. All authors contributed to the writing of the manuscript. 
1017 Table 1: Structure of the sub-networks

\begin{tabular}{lllllll}
\hline & Links per species & $\begin{array}{l}\text { Asymmetry } \\
(\boldsymbol{A})\end{array}$ & $\begin{array}{l}\text { Connectance } \\
(\boldsymbol{C})\end{array}$ & $\begin{array}{l}\text { Nestedness } \\
(\boldsymbol{N})\end{array}$ & $\begin{array}{l}\text { Generality } \\
(\boldsymbol{G}) \boldsymbol{\Psi}\end{array}$ & $\begin{array}{l}\text { Vulnerability } \\
(\boldsymbol{V}) \boldsymbol{\Psi}\end{array}$ \\
\hline Bat-Parasite & 1.02 & $-0.31^{*}$ & 0.09 & 12.60 & $1.95^{*}$ & $1.32^{*}$ \\
Bat-Plant & 1.37 & -0.33 & 0.21 & 26.25 & 2.74 & 1.80 \\
Bat-Prey & 1.70 & -0.97 & 0.16 & 14.11 & 76.72 & 1.52 \\
Bat-BIN & 1.17 & -0.91 & 0.12 & 14.79 & 41.25 & 1.37
\end{tabular}

1018

1019

*see Supplemental Information for an interpretation of positive vs. negative values and structural arrangement

1020

1021

1022

1023 
1024 Table 2: A comparison of three data types for network analysis

\begin{tabular}{|c|c|c|c|c|c|c|c|}
\hline & Data Type & $\begin{array}{l}\text { Taxonomic } \\
\text { Resolution }\end{array}$ & Application & $\begin{array}{l}\text { Required } \\
\text { References }\end{array}$ & Advantages & Disadvantages & $\begin{array}{l}\text { Network } \\
\text { Implications }\end{array}$ \\
\hline $\begin{array}{l}\text { Resolving ID } \\
\text { with DNA } \\
\text {-The plant } \\
\text { network }\end{array}$ & $\begin{array}{l}\text { A few } \\
\text { sequences per } \\
\text { individual }\end{array}$ & Species & $\begin{array}{l}\text { Identification of } \\
\text { fragments }\end{array}$ & $\begin{array}{l}\text { Complete } \\
\text { database }\end{array}$ & $\begin{array}{l}\text {-can deal with forensic trace } \\
\text { material } \\
\text {-produces traditional networks } \\
\text {-minimal taxonomic expertise }\end{array}$ & $\begin{array}{l}\text { - requires molecular } \\
\text { expertise } \\
\text { - requires a well resolved } \\
\text { database }\end{array}$ & $\begin{array}{l}\text {-Produces a network equivalent } \\
\text { to a traditional food web }\end{array}$ \\
\hline $\begin{array}{l}\text { Resolving species } \\
\text { when taxonomy is } \\
\text { not known } \\
\text { - The parasite } \\
\text { network }\end{array}$ & $\begin{array}{l}\text { A few } \\
\text { sequences per } \\
\text { individual }\end{array}$ & $\begin{array}{l}\text { Species but } \\
\text { without names }\end{array}$ & $\begin{array}{l}\text { Identification of taxa } \\
\text { where taxonomy } \\
\text { may be incomplete } \\
\text { or cryptic }\end{array}$ & $\begin{array}{l}\text { Incomplete } \\
\text { database }\end{array}$ & $\begin{array}{l}\text {-can deal with forensic trace } \\
\text { material } \\
\text {-can include species with } \\
\text { incomplete taxonomic } \\
\text { investigation } \\
\text { - resolves cryptic taxa }\end{array}$ & $\begin{array}{l}\text { requires molecular expertise } \\
\text {-may not encourage } \\
\text { taxonomic work }\end{array}$ & $\begin{array}{l}\text {-Similar to a traditional food } \\
\text { web, but may collapse trophic } \\
\text { levels where ID is not clear }\end{array}$ \\
\hline $\begin{array}{l}\text { Using MOTUs } \\
\text { without a } \\
\text { taxonomic unit } \\
\text { - The arthropod } \\
\text { prey network }\end{array}$ & $\begin{array}{l}\text { Millions of } \\
\text { sequences per } \\
\text { sample }\end{array}$ & $\begin{array}{l}\text { Arbitrary but } \\
\text { comparable } \\
\text { units }\end{array}$ & $\begin{array}{l}\text { Rapid surveys where } \\
\text { identification is not } \\
\text { possible }\end{array}$ & $\begin{array}{l}\text { No database } \\
\text { required }\end{array}$ & $\begin{array}{l}\text {-can deal with forensic trace } \\
\text { material } \\
\text {-may include both known and } \\
\text { unknown data (MOTUs) } \\
\text {-can be used in any context } \\
\text { regardless of taxonomic } \\
\text { knowledge } \\
\text {-rapid and MOTUs are } \\
\text { mathematically and genetically } \\
\text { identical yielding perfect } \\
\text { resolution } \\
\text {-quantification is controversial* }\end{array}$ & $\begin{array}{l}\text { - requires molecular } \\
\text { expertise } \\
\text {-does not represent real taxa } \\
\text {-may include error- prone } \\
\text { data } \\
\text {-actual MOTU numbers are } \\
\text { meaningless } \\
\text {-may be biased by primers } \\
\text { or other protocol choices }\end{array}$ & $\begin{array}{l}\text {-Fundamentally different } \\
\text { MOTUs are not species and } \\
\text { likely collapse trophic levels but } \\
\text { allow rapid structural } \\
\text { comparisons } \\
\text {-Node numbers are meaningless }\end{array}$ \\
\hline
\end{tabular}

\footnotetext{
$1025 *$ See a review by Deagle et al. 2018.
} 
Figures:

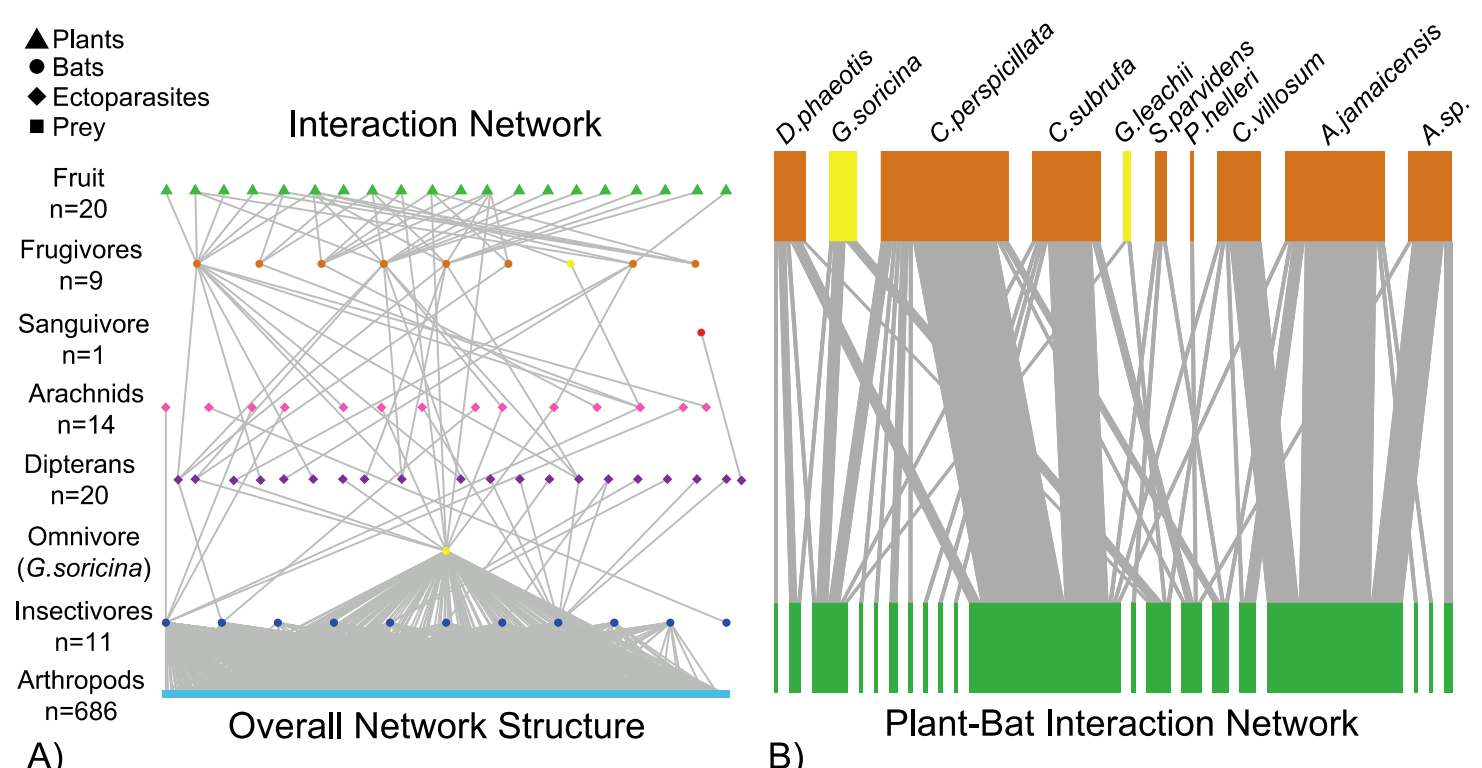

A)

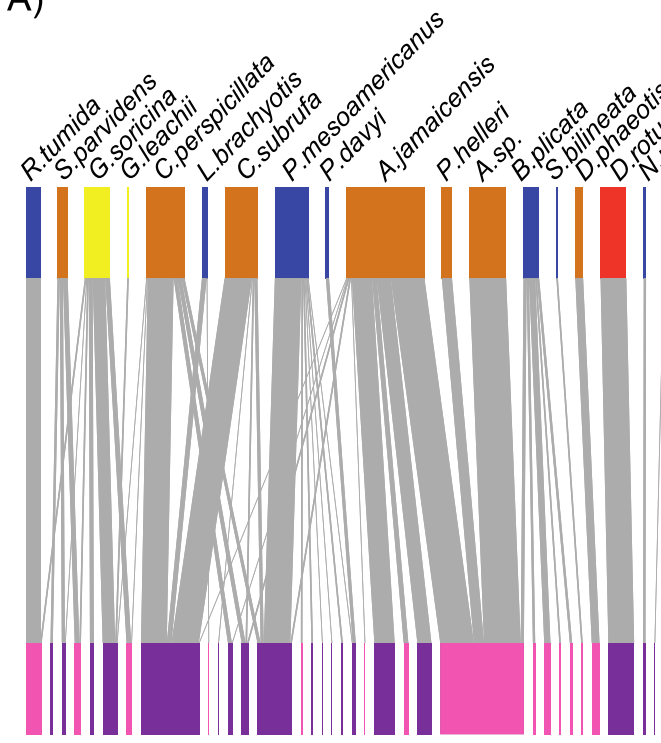

C)

Parasite-Bat Interaction Network

1028

1029

1030

1031

1032

1033

1034

1035

1036

1037

1038

1039

Figure 1: Species' interaction networks. The network of networks (A) displays interaction structure organised by behavioural ecology (rather than traditional trophic structure). The visualization of this network is not presented as standard trophic levels for two reasons. First, the arthropod prey represent multiple trophic levels themselves which cannot be differentiated. Second, the density of connections make links to plants impossible to distinguish if the plants are presented as the lowest trophic level. In this case the network has been structured to depict function rather than trophic levels. For example, arachnid mites of bats are parasites that spend their entire life cycle on their host (Christe, Arlettaz, \& Vogel, 2000) which restricts their dispersal so horizontal transmission primarily occurs via host-to-host contact. Therefore, mites and their hosts are usually regarded as the product of long co-evolution. In comparison, parasitic Diptera (flies) can be highly 
1040 mobile, and often spend part of their life cycle apart from their host (Fritz, 1983). 1041 Because of such distinct life history differences alternative hypotheses of function can be 1042 advanced. We depict them as separate functional groups (A) and in their traditional 1043 parasite role (C). N-values represent the number of taxa detected. Semi-quantified 1044 individual trophic networks (B-D) display traditional trophic organisations (though for 1045 simplicity of comparison bats are always presented on top). Detection frequency data for 1046 each species is given by the width of the block proportional to species' frequency in the 1047 network. Colours indicate behavioural role from A. See Supplemental Information for a 1048 discussion of visualization orientation and Supplemental tables S4 and S8 for matrices of 1049 parasite, plant and bat taxonomic identifications. 

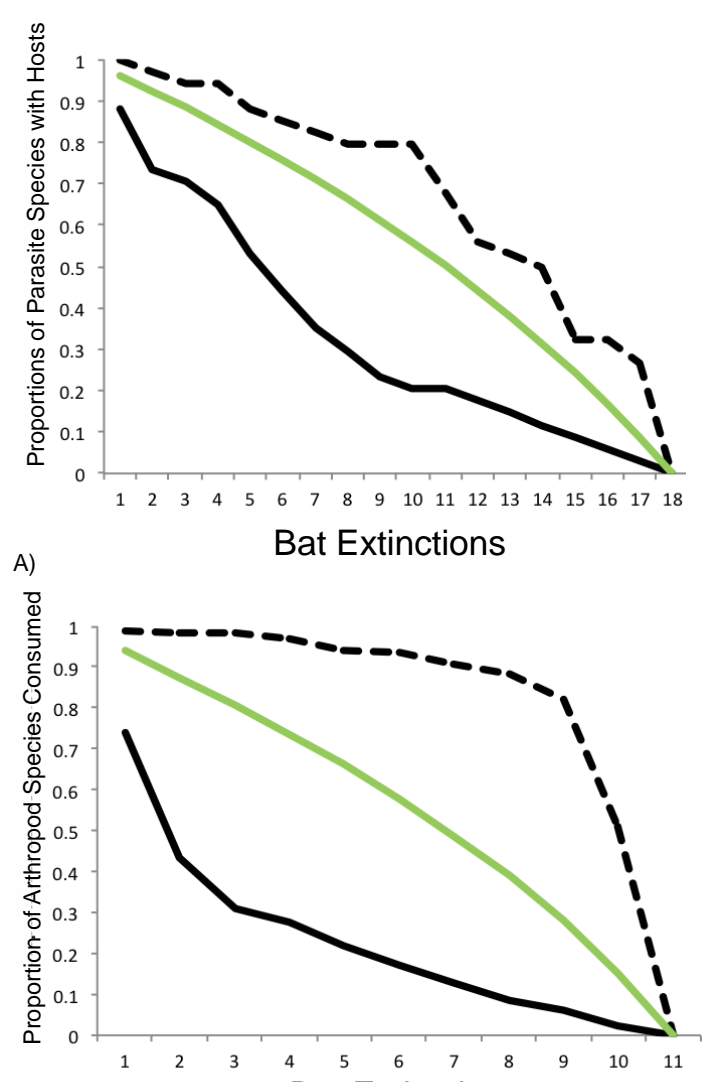

C)

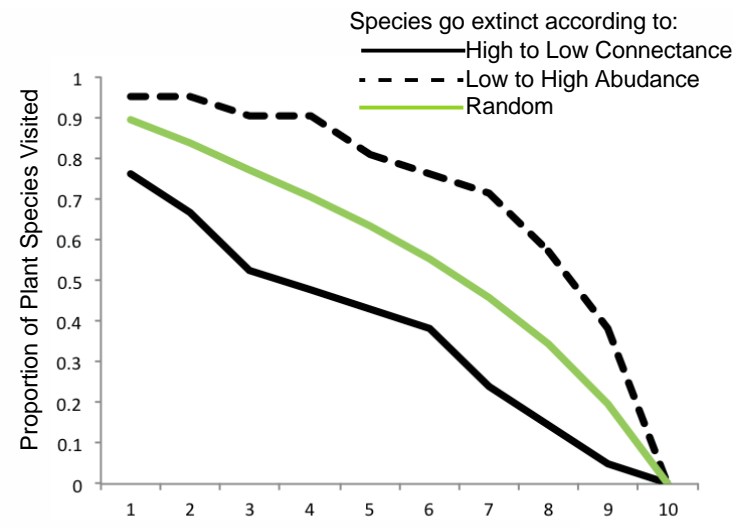

B)

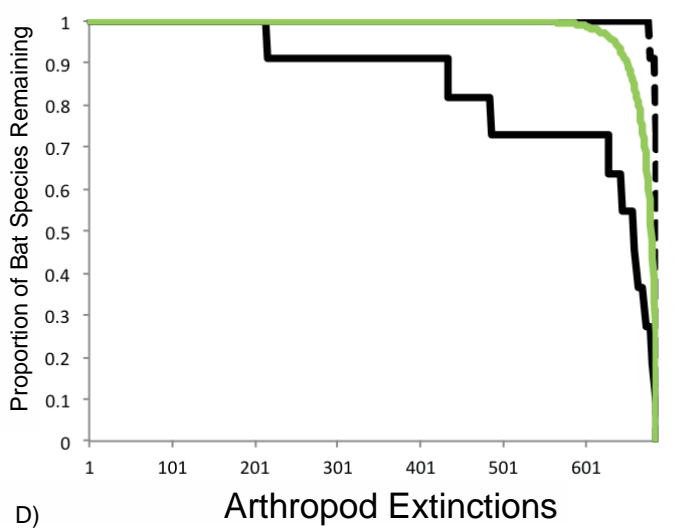

1050

1051

1052

1053

1054

1055

1056

Figure 2: The robustness of interaction network structure to the sequential removal of species under three extinction models. The number of bat species removed has an extreme impact on the loss of parasites (A), while plants are slightly more resilient (B). The proportion of arthropods released from predation (C) is strongly dependent on the model of extinction, while insectivorous bats are extremely resilient to the loss of prey under any model (D). 


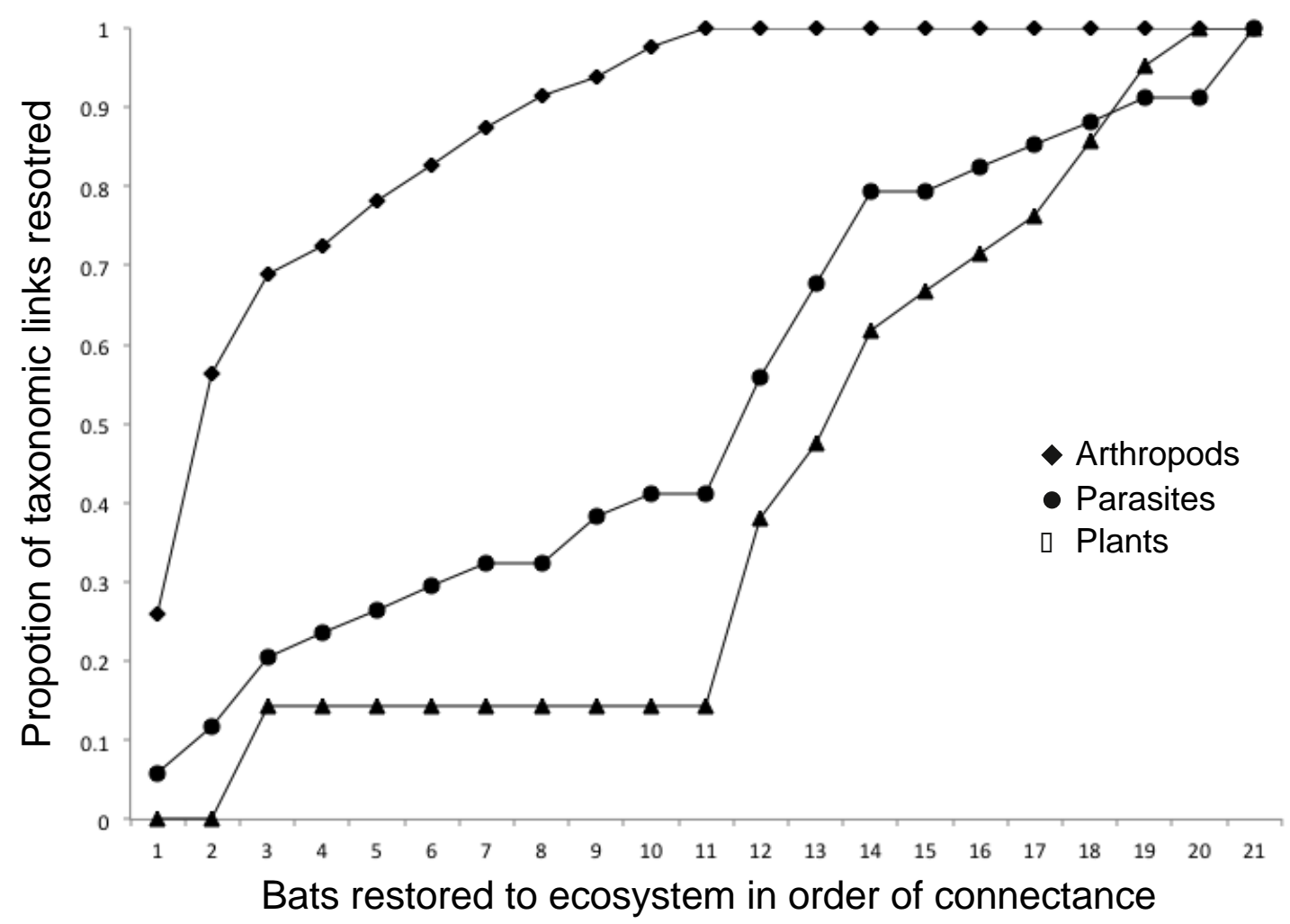

1057

1058

1059

Figure 3: A restoration ecology model showing the proportion of links restored if bats are introduced to the ecosystem in order of connectance (best case scenario). With the 1060 restoration of only the three most strongly connected species (Pteronotus 1061 1062 mesoamericanus, Balantiopteryx plicata and Glossophaga soricina), $72 \%$ of arthropod 1063 species are under predation, $24 \%$ of parasite species have a host, and $14 \%$ of plant 1064 species are visited. See Clare et al., (2014) for a discussion of trophic roles of Glossophaga. 

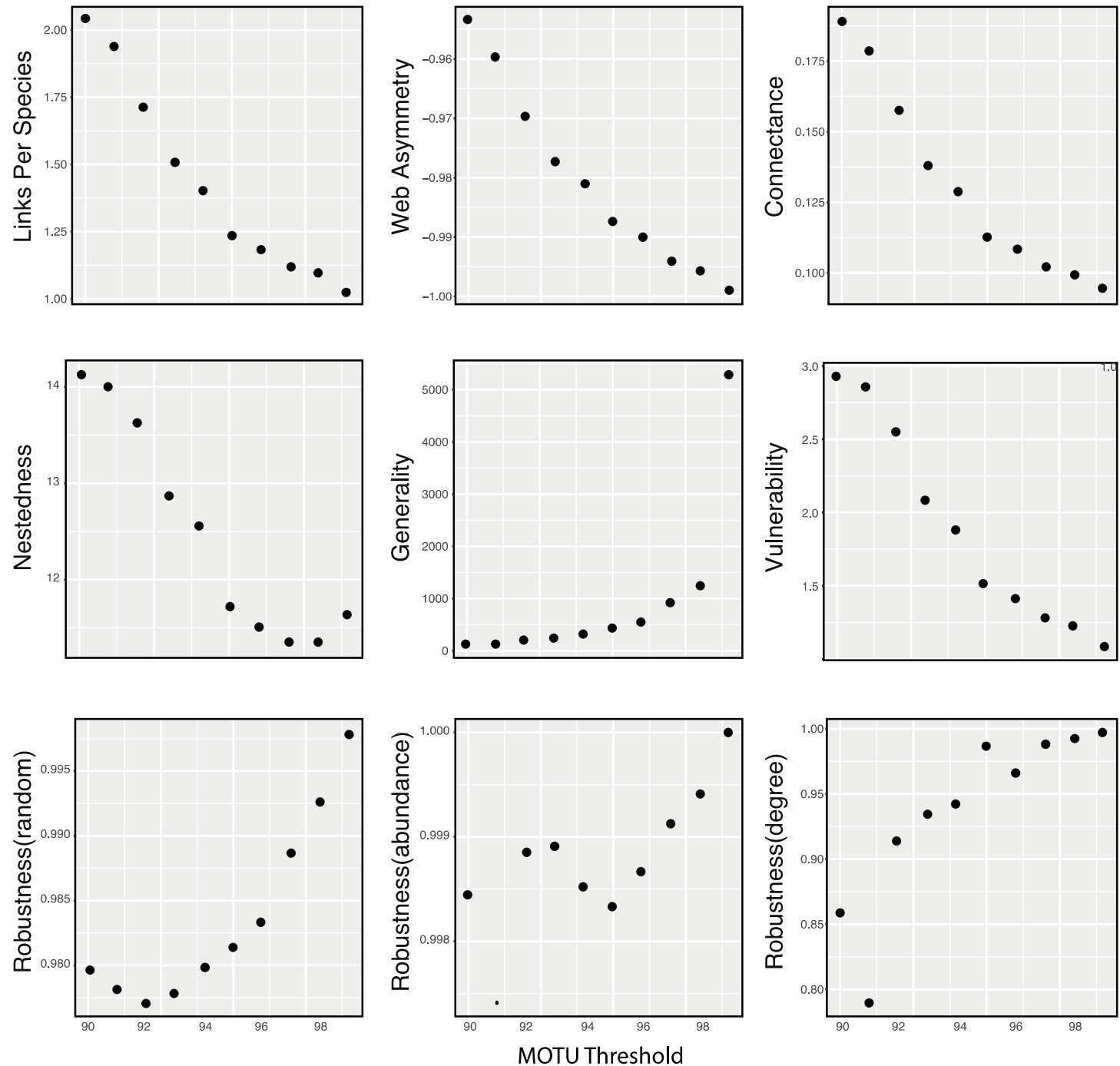

1066

1067

1068

1069

1070

1071

1072

1073

1074

1075

1076

1077

1078

Figure 4: Metabarcode data is a fundamentally new type of data for network ecology. Nodes in metabarcoding normally do not represent a specific taxon or taxonomic level, but are molecular operational taxonomic units (MOTUs) best described as taxa that are defined by being genetically congruent pools of diversity. They are defined by a series of bioinformatics steps with the ultimate decision dependent on the threshold employed for splitting vs. lumping sequences into a MOTU (nodes in our networks). As the MOTU threshold changes, taxa are lumped or split to a greater or lesser extent. For most network metrics (top two rows), this has a predictable effect as the resource level in our networks increases in richness relative to the consumers. The same pattern is evident but less predictable in measures of robustness (bottom row). For a complete analysis see Hemprich-Bennett et al. (2018). 


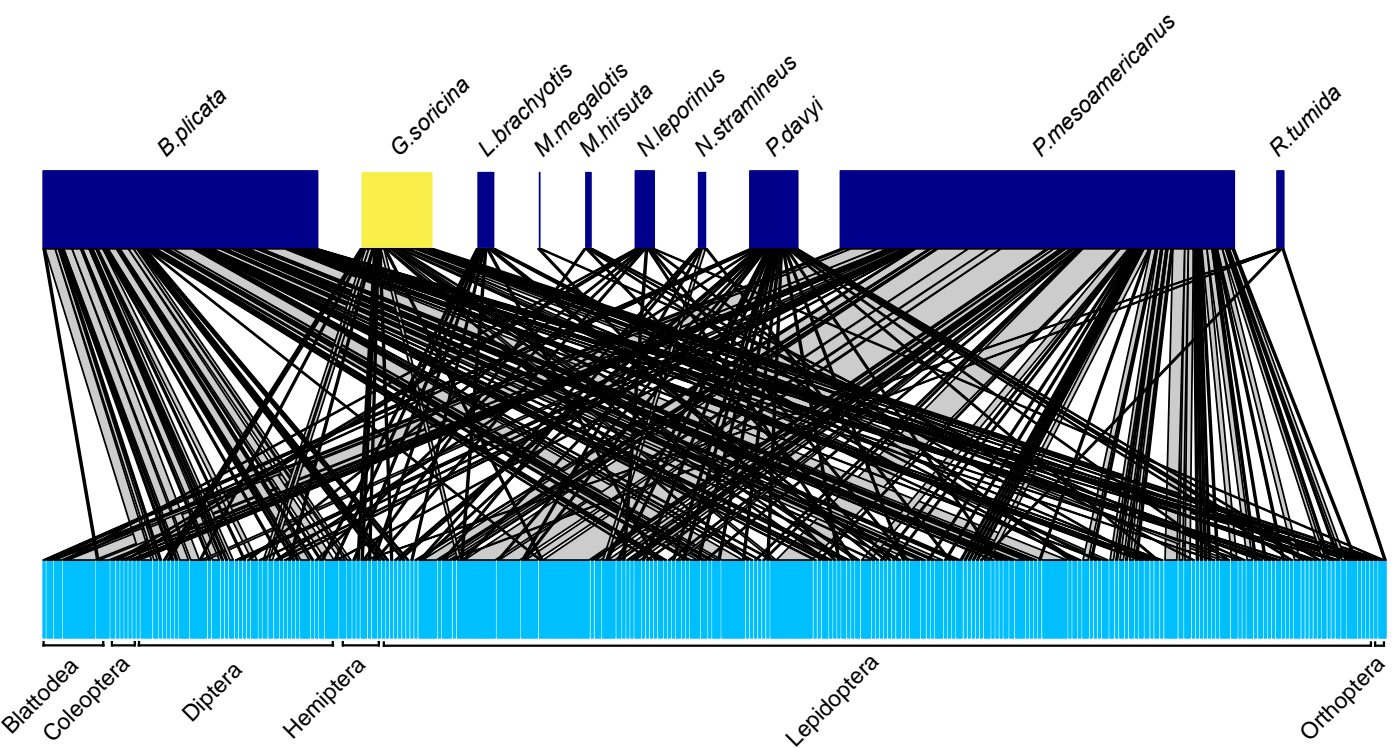

1081

Figure 5: Species' interaction networks for bats and prey identified using the BIN 1082 association method employed on the mBRAVE platform. See table S9 for a matrix of bats and BIN based nodes with full taxonomic identifications. 